\title{
Unified Approach For Multiscale Radar Cross-Section Measurement by Synthetic Aperture Imaging
}

This paper was downloaded from TechRxiv (https://www.techrxiv.org).

\section{LICENSE}

CC BY 4.0

SUBMISSION DATE / POSTED DATE

$26-12-2021$ / 29-12-2021

\section{CITATION}

Watanabe, Takuma; Yamada, Hiroyoshi (2021): Unified Approach For Multiscale Radar Cross-Section Measurement by Synthetic Aperture Imaging. TechRxiv. Preprint.

https://doi.org/10.36227/techrxiv.17693723.v1

$\mathrm{DOI}$

10.36227/techrxiv.17693723.v1 


\title{
Unified Approach For Multiscale Radar Cross-Section Measurement by Synthetic Aperture Imaging
}

\author{
TAKUMA WATANABE ${ }^{1}$ AND HIROYOSHI YAMADA ${ }^{2}$ (MEMBER, IEEE) \\ ${ }^{1}$ Fujitsu Ltd., Kawasaki 2118588, Japan (e-mail: takuma.watanabe.jp@ieee.org) \\ ${ }^{2}$ Faculty of Engineering, Niigata University, Niigata 9502181, Japan. \\ Corresponding author: Takuma Watanabe (e-mail: takuma.watanabe.jp@ieee.org).
}

\begin{abstract}
In this study, we present an improved and unified approach for image-based radar crosssection (RCS) measurement by 2-D synthetic aperture radar (SAR) imaging with an arbitrary curved antenna scanning trajectory. Because RCS is a quantity defined in the far-field distance of an object under test, direct RCS measurement of an electrically large target is often infeasible owing to the spatial limitation of the measurement facility. The method proposed in this study belongs to the class of techniques referred to as the image-based near-field to far-field transformation (NFFFT) to convert the near-field data of scattering experiment into the far-field RCS. In a previous study, we have developed an NFFFT based on 3-D SAR imaging with an arbitrary antenna scanning surface. However, the previous approach is only applicable to the surface scanning which is impossible for a certain case such as measurement using airborne SAR or vehicle-borne SAR. Therefore, one requires an alternative method that can accommodate an arbitrary scanning curve, which is the subject of this study. We derive a generalized correction factor for imagebased NFFFT which is designed to ensure the integral transformation in the image reconstruction process be self-consistent for electrically small scatterers. We provide a series of numerical simulations, an indoor experiment, and an airborne SAR experiment to validate that the proposed scheme can be utilized for various situations ranging from near-field to far-field distance.
\end{abstract}

INDEX TERMS Image reconstruction, near-field to far-field transformation (NFFFT), radar cross-section (RCS), synthetic aperture radar (SAR)

\section{INTRODUCTION}

$\mathbf{R}$ ADAR technologies are becoming more familiar in a wide variety of fields which are not only restricted to defense systems, but also several non-military applications ranging from small- to large-scale problems, and near- to far-field distances; the examples of them include the synthetic aperture radar (SAR) for geoscience and remote sensing, automotive radars, microwave non-destructive testing, medical imaging, radar systems for public security, and so forth. In characterizing an object to be measured by radar systems, radar cross-section (RCS) is one of the fundamental and essential physical parameters, and thus, the demand of accurate RCS prediction and measurement is expected to be continuously increasing. Based on this background, the main objective of this paper is to establish a unified framework for RCS measurement that can accommodate several applications with different scales such as those exemplified before; we refer to this framework as the multiscale RCS measurement in the following discussion.

Because RCS is a quantity defined in the far-field distance which is inversely proportional to an operating wavelength and proportional to a squared dimension of an object under test, direct RCS measurement for an electrically large target such as an aircraft, a ship, and a vehicle using indoor facility is usually infeasible owing to its spatial limitations. Therefore, techniques called the near-field to farfield transformation (NFFFT) have been developed so that one can convert the measured data collected in the nearfield distance to the far-field RCS. The well-known existing NFFFT approaches are: the physical-optics-based method [1], the plane-wave synthesis [2]-[4], and the SAR-based or image-based method [5]-[15] which is focused on this study. In the image-based NFFFT, we first carry out a scattering experiment for a target of interest by an antenna placed in the near-field of the testing object. Then, we reconstruct SAR or inverse SAR (ISAR) image [16]-[19] from the collected data, 
although some methods avoids the explicit image generation [14], [15]. Finally, the obtained image is Fourier-transformed to obtain the far-field RCS. The theoretical background of these processes can be explained as follows: First, we solve the inverse problem of the wave equation under the Born or single-scattering approximation to determine the spatial distribution of the sources of scattering [19], and then we consider solving the forward problem of the wave equation based on the obtained source distribution; if the observation point of this forward problem is assumed to be at infinity (farfield), its solution can be constructed via the inverse Fourier transform of the source distribution (i.e., a SAR or ISAR image) with respect to the spatial variables [8], [13].

Fig. 1 illustrates the concept of the multiscale RCS measurement based on the SAR imaging discussed in this paper. Fig. 1(a) shows typical indoor RCS measurement in an anechoic chamber, where an object under test is mounted on a turntable such that the target is irradiated by an antenna from all azimuthal directions to create a precise circular aperture to form ISAR images. As the range to the target is usually within the near-field distance in this case, application of the NFFFT techniques are essential to obtain the far-field RCS. The merits of the chamber testing are its high motion stability achieved by a turntable, and various source of unwanted backscattering, such as the reflection from the walls, the ground, and other unrelated objects around the target, can be minimized compared to open test sites. However, the feasible dimension of the target is limited by the chamber size and the antenna pattern. Figs. 1(b) and (c) show the outdoor RCS measurement using a vehicle-borne radar and an airborne radar, respectively, where a radar-carrying platform moves along a quasi-circular trajectory instead of rotating the target. These types of SAR observation are well known as the circular SAR (CSAR), and the concept of the CSAR has been demonstrated by indoor experiments [20], [22] and airborne SAR systems [24], [25]. The main advantage of the outdoor measurement is the capability of accommodating a large- and real-scale target without resorting to use of a small scaled model. However, the antenna scanning trajectory is not guaranteed to be an ideal curve such as a circle used in the indoor experiment with a turntable, as the motion of a radarcarrying platform fluctuates depending on several environmental and mechanical conditions such as topography of the test site, weather, wind, and motion stability of the platform. Moreover, unwanted sources of backscattering around the target degrade the accuracy of the RCS measurement.

The aim of this paper is to establish a unified framework of the SAR- or ISAR-based RCS measurement that can handle the different-scale scenarios with various antenna scanning trajectories such as those exemplified in Fig. 1. The approaches based on the SAR or ISAR are known as the image-based NFFFT, although the far-field criterion may be achieved in the medium- or large-scale scenarios shown in Figs. 1(b) and (c). This approach was firstly introduced to convert the near-field data taken in small measurement facilities to far-field RCS, as shown in Fig. 1(a), however, it was proven to be also useful for estimating azimuthal scattering pattern within radar images obtained in the medium- or largescale airborne CSAR scenario shown in Figs. 1(b) and (c) [20].

Although the image-based technique has been proven to be a powerful and reliable tool for RCS measurement, its accuracy is known to be degraded when measuring the target of strong geometric asymmetry in compact facilities, as discussed in [8]. To compensate for this problem, Osipov et al. introduced a correction factor for the 2-D ISAR image reconstruction with a circular scanning geometry [8], and Vaupel extended the concept to a case of 3-D imaging with cylindrical scanning geometry [11], [12]. Based on these works, Watanabe and Yamada derived a generalized correction factor for 3-D synthetic aperture imaging with 2$\mathrm{D}$ antenna scanning along an arbitrary curved surface [13]. However, this generalized correction factor cannot be directly applied to the 1-D antenna scanning along a curved path such as those illustrated in Fig. 1, as the computation involves partial derivatives of the scanning surface with respect to both of two coordinate variables to parametrize the surface. Although the antenna scanning over a surface such as a plane and a cylinder can be readily accomplished using a horizontal-vertical scanner and a turntable in the small-scale scenario presented in Fig. 1(a), this is not the case for vehicleborne or airborne SAR imaging shown in Figs. 1(b) and (c). Therefore, alternative theoretical development is demanded to establish a technique for RCS determination from SAR imaging with an arbitrary curved antenna scanning trajectory. More specifically, this paper proposes a generalized correction factor that can be applied to 2-D SAR or ISAR imaging with a 1-D scanning along an arbitrary trajectory, without requiring 2-D antenna scanning along a surface. By the introduction of this novel correction factor, the imagebased RCS determination is unified such that it can be applied to the multiscale RCS measurement ranging from the smallto large-scale SAR or ISAR scenarios shown in Fig. 1.

The mathematical development of the method with the novel correction factor is based on the previous works presented in [8] and [13], where the former assumes 1-D antenna scanning along a circle as shown in Fig. 1(a), and the latter assumes 2-D scanning along a surface. The present study aims at removing the requirement of the circular or surface scanning, such that RCS can be extracted from a 2D SAR image obtained by vehicle-borne or air-borne radar measurement, as shown in Fig. 1(b) and (c). We discuss some limitations that are introduced in compensation for employing the simpler 2-D SAR imaging instead of the 3$D$ imaging with surface scanning, by a series of numerical simulations using a cloud of point scatterers. Moreover, two types of experimental validation of the proposed method are presented in this study, that is, indoor measurement and airborne circular SAR (CSAR) measurement.

The former experiment corresponds to the small-scale scenario depicted in Fig. 1(a) and its main objective is explicitly showing the improvement in RCS prediction by the proposed 


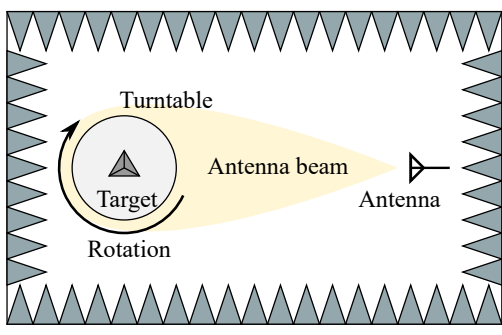

Anechoic chamber

(a)

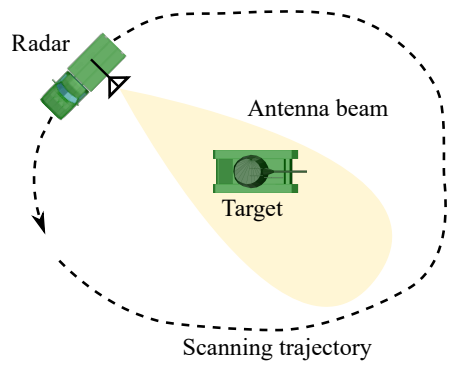

(b)

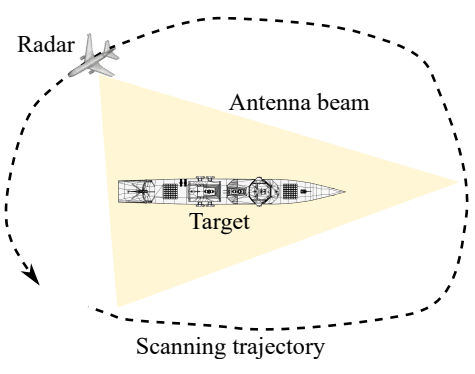

(c)

FIGURE 1. Concept of the multiscale RCS measurement by synthetic aperture imaging with a curved scanning trajectory. This paper proposes a unified framework that can accommodate different-scale scenarios ranging from a small-scale ISAR experiment under an anechoic chamber to a large-scale airborne SAR system. (a) Small-scale scenario in an anechoic chamber. (b) Medium-scale scenario of a vehicle-borne radar system. (c) Large-scale scenario of an airborne SAR system.

correction factor. In this experiment, we consider RCS measurement of a scaled aircraft model made of aluminum in an anechoic chamber. By comparing the experimental results with far-field RCS obtained by a numerical electromagnetic solver, we show that the proposed method with the correction factor predicts far-field RCS of the target more accurately compared to the method without the correction factor. The latter airborne CSAR experiment corresponds to the largescale scenario shown in Fig. 1(b), and our aim is to demonstrate the applicability of the proposed method to a strongly fluctuating antenna trajectory, rather than showing the improvement by the correction factor, because the improvement is not evident as the distance between the antenna and target is sufficiently long in this case. We use a publicly available CSAR dataset (GOTCHA) released by the Air Force Research Laboratory (AFRL) [26]. In this airborne CSAR demonstration, we devise a method for data-driven antenna pattern compensation, because information about the antenna used for this experiment is unavailable from the released dataset.

The major novelty and contribution of this paper are summarized as follows: First, we derive a generalized correction factor for an arbitrary 1-D antenna scanning curve, which is not merely considered to be a special case of the previously derived correction factor for 3-D imaging with 2-D surface scanning [13]. As discussed in Section II-D1, this is a generalization of the correction factor for 2-D circular scanning given in [8]. Second, the proposed image-based method for RCS measurement is validated by the indoor experiment in an anechoic chamber and by the real airborne CSAR measurement. Finally, for the airborne CSAR measurement, a data-driven antenna pattern compensation scheme is proposed to improve the RCS computation. Based on these different-scale experiments, we provide a proof-of-concept that the multiscale RCS measurement is possible within the proposed unified framework.

The remainder of this paper is organized as follows: Section II describes the mathematical development of the problem. The proposed correction factor is discussed in Section
II-C, In Section III, we investigate the proposed method in detail by a series of simple point scatterer simulations. We discuss the improvement by the correction factor and limitations introduced by the 2-D imaging with an arbitrary 1 -D curved scanning. The effect of the correction factor is experimentally validated in Section IV, where the measurement was carried out in an anechoic chamber. We employ a scaled aircraft model made of aluminum as a target for this experiment. Section V shows another experimental validation using the real airborne CSAR dataset. As mentioned before, the purpose of this experiment is to confirm the applicability of the proposed correction factor to an arbitrary scanning curve without its analytical representation, rather than showing the improvement of the RCS prediction, which is proved in Section IV. Finally, Section VI concludes the study presented in this paper and discusses future consideration.

\section{PROBLEM FORMULATION}

In this section, we discuss formulation of the problem. The mathematical development follows those presented in [13], except that we consider the 1-D antenna scanning along a curved path, instead of 2-D scanning along a curved surface. The system and signal model of the problem are discussed first, and the image reconstruction procedure is described. Then, we derive a generalized correction factor for the image reconstruction to improve RCS prediction accuracy, and discuss how this correction factor can be applied to an arbitrary curved 1-D scanning geometry. As the final step, we describe RCS computation from the reconstructed image.

\section{A. SYSTEM AND SIGNAL MODEL}

Fig. 2 illustrates the system model of synthetic aperture imaging with an arbitrary curved antenna scanning trajectory. We consider an object under test placed in 3-D spatial domain, where an arbitrary location in the space is denoted by $\boldsymbol{r}=(x, y, z)$. This target is irradiated by an antenna at $\boldsymbol{r}_{0}(u)$ on an arbitrarily curved scanning trajectory enclosing the object, where the scanning curve is parametrized by the variable $u$. The curve is not necessary be planner or closed such as a circle within $(x, y)$-plane, and the curve can be 


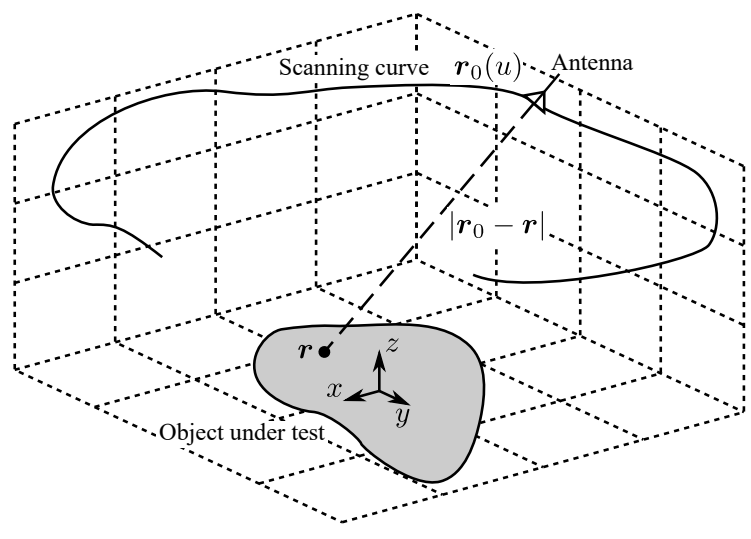

FIGURE 2. System model of synthetic aperture imaging with an arbitrarily curved antenna scanning trajectory.

represented by a discrete set of coordinates without having explicit analytical expression. However, a certain restriction must be imposed on this scanning curve to complete the mathematical development; this point is discussed later in the derivation of the correction factor in Section II-C.

The arbitrary locations $\boldsymbol{r}$ and the scanning curve $\boldsymbol{r}_{0}(u)$ are expressed as follows:

$$
\begin{aligned}
\boldsymbol{r}_{0}(u) & =x_{0}(u) \widehat{\boldsymbol{x}}+y_{0}(u) \widehat{\boldsymbol{y}}+z_{0}(u) \widehat{\boldsymbol{z}} \\
\boldsymbol{r} & =x \widehat{\boldsymbol{x}}+y \widehat{\boldsymbol{y}}+z \widehat{\boldsymbol{z}}
\end{aligned}
$$

where $\widehat{\boldsymbol{x}}, \widehat{\boldsymbol{y}}$, and $\widehat{\boldsymbol{z}}$ are the unit vectors along $x-, y$-, and $z$ direction, respectively. Moreover, we define a vector $\boldsymbol{R}(u)$ from the location $\boldsymbol{r}$ to the antenna location $\boldsymbol{r}_{0}(u)$ on the scanning curve as

$$
\begin{aligned}
\boldsymbol{R}(u) & =\boldsymbol{r}_{0}-\boldsymbol{r}=R_{x} \widehat{\boldsymbol{x}}+R_{y} \widehat{\boldsymbol{y}}+R_{z} \widehat{\boldsymbol{z}} \\
R_{x}(u) & =x_{0}(u)-x \\
R_{y}(u) & =y_{0}(u)-y \\
R_{z}(u) & =z_{0}(u)-z .
\end{aligned}
$$

Using the vector $\boldsymbol{R}$, we can calculate the distance between the arbitrary location $\boldsymbol{r}$ and the antenna location $\boldsymbol{r}_{0}(u)$, as follows:

$$
R(u)=|\boldsymbol{R}(u)|=\left|\boldsymbol{r}_{0}-\boldsymbol{r}\right|=\sqrt{R_{x}^{2}+R_{y}^{2}+R_{z}^{2}} .
$$

We consider a monostatic scattering experiment under the aforementioned configuration. The antenna at $\boldsymbol{r}_{0}(u)$ transmits an electromagnetic wave of an angular frequency $\omega$, and collects the scattered waves from the object. We denote the propagation speed of the electromagnetic wave by $c$, and define the wavenumber as $k=\omega / c$.

Next, we develop a model to describe the backscattering from the object under test. Let us consider a point scatterer with the reflection coefficient $C$ located at $\boldsymbol{r}$. For this discrete scatterer, the received signal $E^{s}\left(k, \boldsymbol{r}_{0}\right)$ at the antenna location $\boldsymbol{r}_{0}(u)$ can be expressed as follows:

$$
E^{s}\left(k, \boldsymbol{r}_{0}\right)=P^{2}\left(k, \boldsymbol{r}_{0}, \boldsymbol{r}\right) \frac{k^{2} C}{\sqrt{4 \pi}} \frac{e^{-2 j k\left|\boldsymbol{r}_{0}-\boldsymbol{r}\right|}}{\left|\boldsymbol{r}_{0}-\boldsymbol{r}\right|^{2}}
$$

where $P\left(k, \boldsymbol{r}_{0}, \boldsymbol{r}\right)$ represents the antenna pattern, assuming that the transmitting and the receiving antenna are collocated and the radiation patterns are same.

The signal model of Eq. (4) can be generalized to a case of distributed scatterers, where the reflection coefficient at the location $\boldsymbol{r}$ is denoted by $C(\boldsymbol{r})$. The received signal for this case is given by

$$
E^{s}\left(k, \boldsymbol{r}_{0}\right)=\frac{k^{2}}{\sqrt{4 \pi}} \int_{V} P^{2}\left(k, \boldsymbol{r}_{0}, \boldsymbol{r}\right) C(\boldsymbol{r}) \frac{e^{-2 j k\left|\boldsymbol{r}_{0}-\boldsymbol{r}\right|}}{\left|\boldsymbol{r}_{0}-\boldsymbol{r}\right|^{2}} .
$$

If the object can be regarded as a cloud of point scatterers, the reflection coefficient $C(\boldsymbol{r})$ is expressed as

$$
C(\boldsymbol{r})=\sum_{i} C_{i} \delta\left(\left|\boldsymbol{r}_{0}-\boldsymbol{r}_{i}\right|\right)
$$

where $C_{i}$ is the reflection coefficient of the $i$ th point scatterer. If the scatter is a small conducting sphere of radius $a_{i}\left(k a_{i}<\right.$ 0.4 ), the coefficient $C_{i}$ can be given by [8], [27]

$$
C_{i}=3 \sqrt{\pi} a_{i}^{3}
$$

Plugging Eq. (6) into Eq. (5) results in the following discrete scatterer model:

$$
E^{s}\left(k, \boldsymbol{r}_{0}\right)=\frac{k^{2}}{\sqrt{4 \pi}} \sum_{i} P^{2}\left(k, \boldsymbol{r}_{0}, \boldsymbol{r}_{i}\right) C_{i} \frac{e^{-2 j k\left|\boldsymbol{r}_{0}-\boldsymbol{r}_{i}\right|}}{\left|\boldsymbol{r}_{0}-\boldsymbol{r}_{i}\right|^{2}} .
$$

We use the discrete scatterer model of Eq. (8) to test the proposed image-based RCS measurement in Section III.

\section{B. IMAGE RECONSTRUCTION}

For the image-based RCS determination, we need to first reconstruct a spatial image from the collected received signal $E^{s}\left(k, \boldsymbol{r}_{0}\right)$. We consider 2-D image reconstruction within $(x, y)$-plane at a constant altitude $z_{c}$; we refer to this $(x, y)$ plane as image plane in the following discussion. If a single point scatterer at $\left(x_{1}, y_{1}, z_{1}\right)$ with reflection coefficient $C_{1}$ is considered, an ideal spatial image is given by

$$
\psi(\boldsymbol{r})=C_{1} \delta\left(x-x_{1}\right) \delta\left(y-y_{1}\right)
$$

where $\delta(\cdot)$ is the delta function. In the following, our aim is to recover the ideal image given by Eq. (9) from the received signal $E^{s}\left(k, \boldsymbol{r}_{0}\right)$.

The image reconstruction is generally expressed as the following integral transformation of the received signal:

$$
\psi(\boldsymbol{r})=\int_{0}^{\infty} \int_{D_{u}} E^{s}\left(k, \boldsymbol{r}_{0}\right) F\left(k, \boldsymbol{r}_{0}, \boldsymbol{r}\right) d u d k
$$

where $D_{u}$ is the domain of the integration regarding $u$, and $F\left(k, \boldsymbol{r}_{0}, \boldsymbol{r}\right)$ is a weighting function called a focusing factor given by

$$
F\left(k, \boldsymbol{r}_{0}, \boldsymbol{r}\right)=g\left(k, \boldsymbol{r}_{0}, \boldsymbol{r}\right) \frac{\left|\boldsymbol{r}_{0}-\boldsymbol{r}\right|^{2}}{P^{2}\left(k, \boldsymbol{r}_{0}, \boldsymbol{r}\right)} e^{2 j k\left|\boldsymbol{r}_{0}-\boldsymbol{r}\right|}
$$

where $g\left(k, \boldsymbol{r}_{0}, \boldsymbol{r}\right)$ is a correction factor to be derived. Note that $g\left(k, \boldsymbol{r}_{0}, \boldsymbol{r}\right)=1$ corresponds to the standard SAR or ISAR formulation. 
We can numerically implement Eq. (10) by replacing the integrals in Eq. (10) with the following summations:

$$
\psi(\boldsymbol{r}) \simeq \sum_{\kappa} \sum_{m} E^{s}\left[k_{\kappa}, \boldsymbol{r}_{0}\left(u_{m}\right)\right] F\left[k_{\kappa}, \boldsymbol{r}_{0}\left(u_{m}\right), \boldsymbol{r}\right]
$$

where $k_{\kappa}$ and $u_{m}$ are the discretized values of $k$ and $u ; \kappa$ and $m$ are indices representing each sample.

\section{CORRECTION FACTOR}

The correction factor $g\left(k, \boldsymbol{r}_{0}, \boldsymbol{r}\right)$ must be determined such that the integral transformation defined in Eq. (10) be selfconsistent for for an electrically small scatterer [8]; that is, we determine the correction factor $g\left(k, \boldsymbol{r}_{0}, \boldsymbol{r}\right)$ such that Eq. (10) recovers the ideal spatial image of Eq. (9). According to the Eq. (4), we consider the received signal of a point scatterer located at $\boldsymbol{r}_{1}$, as follows:

$$
E_{1}^{s}\left(k, \boldsymbol{r}_{0}\right)=P^{2}\left(k, \boldsymbol{r}_{0}, \boldsymbol{r}_{1}\right) \frac{k^{2} C_{1}}{\sqrt{4 \pi}} \frac{e^{-2 j k\left|\boldsymbol{r}_{0}-\boldsymbol{r}_{1}\right|}}{\left|\boldsymbol{r}_{0}-\boldsymbol{r}_{1}\right|^{2}}
$$

Plugging the received signal of Eq. (13) into Eq. (10) yields the following expression:

$$
\begin{array}{r}
\psi(\boldsymbol{r})=\frac{C_{1}}{\sqrt{4 \pi}} \int_{0}^{\infty} \int_{D_{u}} g\left(k, \boldsymbol{r}_{0}, \boldsymbol{r}\right) \frac{P^{2}\left(k, \boldsymbol{r}_{0}, \boldsymbol{r}_{1}\right)}{P^{2}\left(k, \boldsymbol{r}_{0}, \boldsymbol{r}\right)} \frac{\left|\boldsymbol{r}_{0}-\boldsymbol{r}\right|^{2}}{\left|\boldsymbol{r}_{0}-\boldsymbol{r}_{1}\right|^{2}} \\
\cdot e^{j 2 k s} k^{2} d u d k \\
s=\left|\boldsymbol{r}_{0}-\boldsymbol{r}\right|-\left|\boldsymbol{r}_{0}-\boldsymbol{r}_{1}\right|
\end{array}
$$

As shown in Fig. 3, we define a local coordinate system centered at the scatterer location $\boldsymbol{r}_{1}$. In this local coordinate system, the local azimuthal angle $\alpha(u)$ and zenith angle $\beta(u)$ of the vector $\boldsymbol{R}$ is expressed by

$$
\begin{aligned}
\alpha(u) & =\tan ^{-1}\left[R_{y}(u) / R_{x}(u)\right] \\
\beta(u) & =\tan ^{-1}\left[\rho(u) / R_{z}(u)\right] \\
\rho(u) & =\sqrt{x_{0}^{\prime 2}(u)+y_{0}^{\prime 2}(u)}
\end{aligned}
$$

Using the angles $\alpha(u)$ and $\beta(u)$, the function $s$ can be approximated in a vicinity of the scatterer (i.e., $\boldsymbol{r} \rightarrow \boldsymbol{r}_{1}$ ) as follows [8], [11], [12]:

$$
\begin{aligned}
s \approx\left(x-x_{1}\right) \sin \beta \cos \alpha & +\left(y-y_{1}\right) \sin \beta \sin \alpha \\
& +\left(z-z_{1}\right) \cos \beta
\end{aligned}
$$

Substituting (16) into (14) yields the following expression:

$$
\begin{aligned}
\psi(\boldsymbol{r})= & \frac{C_{1}}{\sqrt{4 \pi}} \int_{0}^{\infty} \int_{D_{u}} g\left(k, \boldsymbol{r}_{0}, \boldsymbol{r}\right) \\
& \cdot \exp \left[j 2 k\left(x-x_{1}\right) \sin \beta \cos \alpha\right] \\
& \cdot \exp \left[j 2 k\left(y-y_{1}\right) \sin \beta \sin \alpha\right] \\
& \cdot \exp \left[j 2 k\left(z-z_{1}\right) \cos \beta\right] k^{2} d u d k
\end{aligned}
$$

In the double integral defined by Eq. (17), we consider the following transformation of the variables:

$$
\begin{aligned}
k_{x} & =2 k \sin \beta \cos \alpha \\
k_{y} & =2 k \sin \beta \sin \alpha \\
k_{z} & =2 k \cos \beta
\end{aligned}
$$

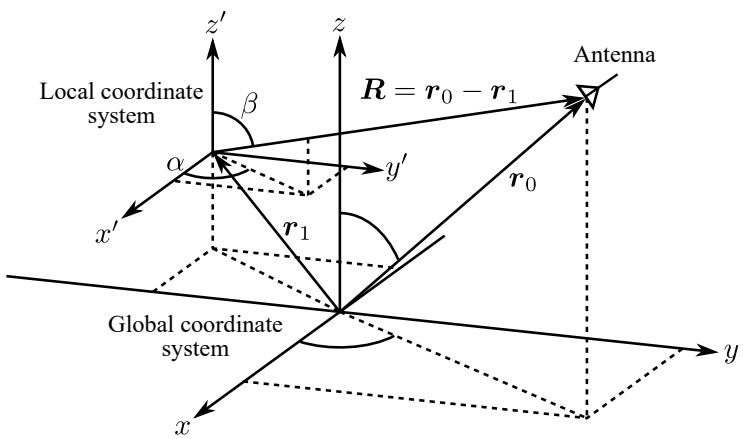

FIGURE 3. Local coordinate system centered at the location $\boldsymbol{r}_{1}$.

where $k_{x}, k_{y}$, and $k_{z}$ corresponds to the spatial frequencies with respect to $x^{\prime}-, y^{\prime}$-, and $z^{\prime}$-direction, respectively, and $k_{z}$ depends on $\left(k_{x}, k_{y}\right)$; these spatial frequencies are defined in the local coordinate system centered at the point $\boldsymbol{r}$. As mentioned earlier, some restrictions must be imposed on the scanning curve $\boldsymbol{r}_{0}(u)$; that is, here we assume that the scanning curve is closed and the range of the local azimuthal is $[0,2 \pi]$ for every scatterers within the area to be imaged. If the scanning trajectory is an open curve, we can virtually extend that curve such that it becomes a closed curve by considering the received signal at the extended part to be zero; under this modification, the similar discussion as the closed curve is established for the open curve. Moreover, every points on the scanning curve $\boldsymbol{r}_{0}(u)$ can be uniquely specified by the local azimuthal angle $\alpha$.

Since we have assumed that the range of the azimuthal angle $\alpha$ is $[0,2 \pi]$, the domains of the integration with respect to the spatial frequencies $k_{x}$ and $k_{y}$ are both $[-\infty, \infty]$. Therefore, substituting Eq. (18) in to Eq. (17) yields the following expression:

$$
\begin{aligned}
\psi(\boldsymbol{r})= & \frac{C_{1}}{\sqrt{4 \pi}} \int_{-\infty}^{\infty} \int_{-\infty}^{\infty} g\left(k, \boldsymbol{r}_{0}, \boldsymbol{r}\right) \\
& \cdot e^{j k_{x}\left(x-x_{1}\right)+j k_{y}\left(y-y_{1}\right)+j k_{z}\left(z-z_{1}\right)} \frac{k^{2}}{|J|} d k_{x} d k_{y}
\end{aligned}
$$

where $J$ represents the Jacobian defined as

$$
J=\frac{\partial\left(k_{x}, k_{y}\right)}{\partial(k, u)}=\left|\begin{array}{ll}
\frac{\partial k_{x}}{\partial k} & \frac{\partial k_{x}}{\partial u} \\
\frac{\partial k_{y}}{\partial k} & \frac{\partial k_{y}}{\partial u}
\end{array}\right|=\frac{\partial k_{x}}{\partial k} \frac{\partial k_{y}}{\partial u}-\frac{\partial k_{x}}{\partial u} \frac{\partial k_{y}}{\partial k} .
$$

The elements of the Jacobian $J$ can be calculated as

$$
\begin{aligned}
& \frac{\partial k_{x}}{\partial k}=2 \cos \alpha \sin \beta \\
& \frac{\partial k_{x}}{\partial u}=2 k\left(\beta_{u} \cos \alpha \cos \beta-\alpha_{u} \sin \alpha \sin \beta\right) \\
& \frac{\partial k_{y}}{\partial k}=2 \sin \alpha \sin \beta \\
& \frac{\partial k_{y}}{\partial u}=2 k\left(\beta_{u} \cos \alpha \cos \beta+\alpha_{u} \sin \alpha \sin \beta\right)
\end{aligned}
$$


where we have used the following definitions:

$$
\begin{aligned}
& \alpha_{u}=\frac{1}{\rho^{2}}\left(R_{x} y_{0 u}-R_{y} x_{0 u}\right) \\
& \beta_{u}=\frac{1}{\rho r_{0}^{\prime 2}}\left[R_{z}\left(R_{x} x_{0 u}+R_{y} y_{0 u}\right)-\rho^{2} R_{z}\right]
\end{aligned}
$$

where $\alpha_{u}$ and $\beta_{u}$ represent the derivative of $\alpha$ and $\beta$ with respect to $u$, respectively.

For a simple antenna scanning curve given by an analytical expression (e.g., a straight line, a circle, and an ellipse), exact expressions for the derivatives $\alpha_{u}$ and $\beta_{u}$ can be derived, as discussed in Section II-D. Otherwise, we can evaluate these quantities by numerical differentiation; we deal with this case in Section II-E.

From Eq. (21), the Jacobian defined in Eq. (20) results in

$$
J=4 k \alpha_{u} \sin ^{2} \beta=\frac{1}{\rho^{2}} 4 k\left(R_{x} y_{0 u}-R_{y} x_{0 u}\right) \sin ^{2} \beta .
$$

Therefore, by selecting the correction factor $g\left(k, \boldsymbol{r}_{0}, \boldsymbol{r}\right)$ to be

$$
\begin{aligned}
g\left(k, \boldsymbol{r}_{0}, \boldsymbol{r}\right) & =\frac{|J|}{2 \pi^{\frac{3}{2}} k^{2}} e^{-j k_{z}\left(z-z_{1}\right)} \\
& =\frac{2}{\pi^{\frac{3}{2}} k}\left|\alpha_{u}\right| \sin ^{2} \beta e^{-j k_{z}\left(z-z_{1}\right)},
\end{aligned}
$$

and comparing Eq. (24) with the integral representation of the 2-D delta function

$$
\begin{gathered}
\frac{1}{(2 \pi)^{2}} \int_{-\infty}^{\infty} \int_{-\infty}^{\infty} e^{j k_{x}\left(x-x_{1}\right)+j k_{y}\left(y-y_{1}\right)} d k_{x} d k_{y} \\
=\delta\left(x-x_{1}\right) \delta\left(y-y_{1}\right),
\end{gathered}
$$

we confirm that Eq. (19) reduces to Eq. (9); that is, the desired image reconstruction is accomplished.

However, as the correction factor $g\left(k, \boldsymbol{r}_{0}, \boldsymbol{r}\right)$ in Eq. (24) contains the unknown altitude of the scatter $z_{1}$, a certain assumption has to be made to evaluate these factors. Therefore, we assume that the the altitude of the scatterer is close to the image plane; that is, we assume $z_{1} \rightarrow z$. This assumption means that we have knowledge about the scatterer altitude, and the scatterer is considered to be sufficiently thin. If this assumption is infeasible, the antenna must be scanned on a 3-D surface such as a cylinder and a sphere, as discussed in [11]-[13]. The effect of this scatterer altitude is investigated in detail in Section III-C.

Therefore, the final forms of the correction factor is defined as follows:

$$
g\left(k, \boldsymbol{r}_{0}, \boldsymbol{r}\right)=\frac{2}{\pi^{\frac{3}{2}} k}\left|\alpha_{u}\right| \sin ^{2} \beta
$$

The derived correction factor given in Eq. (26) has similar form as those derived in [8], except for the factor $\sin ^{2} \beta$. If the altitudes of the antenna and the scatterer are equal, Eq. (26) reduces to the exactly same form given in [8]. However, the correction factor derived in this study is more general in that it accounts for nonzero antenna altitude, and the factor $\alpha_{u}$ can be computed for an arbitrary curved antenna trajectory. When a simple analytical expression for a scanning curve is available, we can exactly evaluate $\alpha_{u}$; this case is exemplified

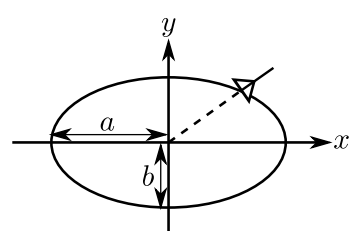

(a) (b)

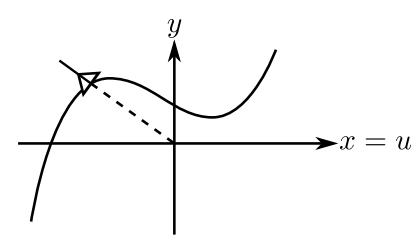

FIGURE 4. Canonical antenna scanning curves. These curves are assumed to be planar curves within $(x, y)$-plane at a constant altitude $z_{0}$. (a) Ellipse. (b) Polynomial curve.

in Section II-D. Even if an analytical formula for a scanning curve is unavailable, we can compute $\alpha_{u}$ by numerical differentiation, as discussed in Section II-E.

\section{EXACT CORRECTION FACTORS FOR SPECIAL CURVES}

In this section, we derive the exact correction factor defined in Eq. (26) for a canonical antenna scanning curve for which an analytical expression is available. We consider two examples of antenna scanning curves depicted in Fig. 4, where (a) and (b) are an ellipse and a polynomial curve, respectively. For simplicity, these curves are assumed to be planar curves within $(x, y)$-plane at a constant altitude $z_{0}$.

\section{1) Ellipses}

Fig. 4(a) shows an ellipse with the semi-major axis $a$ and the semi-minor axis $b$. This ellipse is defined as the following expressions:

$$
x_{0}(u)=a \cos u, \quad y_{0}(u)=b \sin u, \quad z_{0}(u)=z_{0}
$$

Note that a circular aperture corresponds to $a=b$. Using Eq. (27), Eq. (22a) can be evaluated as

$$
\alpha_{u}=\rho^{-2}\left(R_{x} b \cos u+R_{y} a \sin u\right) .
$$

Therefore, the correction factor for this ellipse is given by

$$
g\left(k, \boldsymbol{r}_{0}, \boldsymbol{r}\right)=\frac{2 \sin ^{2} \beta}{\pi^{3 / 2} k \rho^{2}}\left|R_{x} b \cos u+R_{y} a \sin u\right| .
$$

When a circular scanning curve of radius $a$ is considered, Eq. (29) can be written as follows:

$$
\begin{aligned}
g\left(k, \boldsymbol{r}_{0}, \boldsymbol{r}\right) & =\frac{2 a \sin ^{2} \beta}{\pi^{\frac{3}{2}} k \rho^{2}}\left|R_{x} \cos u+R_{y} \sin u\right| \\
& =\frac{2 a^{2} \sin ^{2} \beta}{\pi^{\frac{3}{2}} k \rho^{2}}\left|1-\frac{1}{a}(x \cos u+y \sin u)\right| \\
& =\frac{2 a^{2} \sin ^{2} \beta}{\pi^{\frac{3}{2}} k \rho^{2}}\left[1-\frac{\widehat{\rho}}{a} \cos (\widehat{u}-u)\right]
\end{aligned}
$$

where

$$
\widehat{\rho}=\sqrt{x^{2}+y^{2}}, \quad \widehat{u}=\tan ^{-1}(y / x) .
$$

If the altitudes of the antenna and the image plane are same, the local zenith angle is $\beta=\pi / 2$ and Eq. (30) is further simplified to

$$
g\left(k, \boldsymbol{r}_{0}, \boldsymbol{r}\right)=\frac{2 a^{2}}{k \pi^{\frac{3}{2}} \rho^{2}}\left[1-\frac{\widehat{\rho}}{a} \cos (\widehat{u}-u)\right]
$$


which is the exactly same correction factor as those given in [8]; therefore, Eq. (29) is a generalization of the conventional correction factor for a circular scanning.

\section{2) Polynomial Curves}

Next, we consider a polynomial curve shown in Fig. 4(b). As an example, we define the following polynomial expression:

$$
x_{0}(u)=u, \quad y_{0}(u)=\sum_{n=0}^{N} a_{n} u^{n}, \quad z_{0}(u)=z_{0}
$$

where $a_{n}$ is a known $n$th order polynomial coefficient, and $N$ is the maximum order; a linear aperture corresponds to $N=1$. Using Eq. (33), Eq. (22a) is calculated as

$$
\alpha_{u}=\frac{1}{\rho^{2}}\left(R_{x} \sum_{n=1}^{N} n a_{n} u^{n-1}-R_{y}\right) .
$$

Thus, the correction factor for this polynomial curve is

$$
g\left(k, \boldsymbol{r}_{0}, \boldsymbol{r}\right)=\frac{2 \sin ^{2} \beta}{\pi^{3 / 2} k \rho^{2}}\left|R_{x} \sum_{n=1}^{N} n a_{n} u^{n-1}-R_{y}\right| .
$$

If a linear synthetic aperture is considered, which is the usual case in airborne or spaceborne SAR systems, Eq. (35) reduces to

$$
g\left(k, \boldsymbol{r}_{0}, \boldsymbol{r}\right)=\frac{2 \sin ^{2} \beta}{\pi^{3 / 2} k \rho^{2}}\left|R_{x} a_{1}-R_{y}\right| .
$$

The aforementioned polynomial representation of a scanning curve is important in that it serves as the basis of the numerical correction factor for an arbitrary scanning curve, which is discussed in Section II-E in detail.

\section{E. NUMERICAL CORRECTION FACTOR}

As discussed in Section II-D, the computation of the correction factor requires the differential coefficients of the scanning curves, that is, $x_{0 u}$ and $y_{0 u}$. However, an analytical expression of the scanning curve may unavailable for a certain situation, such as the airborne CSAR measurement discussed in Section V. In this case, we must numerically evaluate the derivatives of the scanning curves. This concept, referred to as the numerical correction factor, was firstly introduced for an arbitrary scanning surface in [13]. In the following, we provide the numerical correction factor specially tailored for an arbitrary curved scanning trajectory.

As shown in Fig. 5, we consider a case in which a scanning curve is given by a set of $M$ discrete sample points as $\boldsymbol{r}_{0}\left(u_{m}\right), m \in\{1,2, \ldots, M\}$. Note that an analytical expression for this curve is unnecessary. Our aim is to compute the derivative of this discrete curve at each sample point $u_{m}$ numerically. In the following discussion, we consider two different types of numerical differentiation, namely, the finite difference approximation and the polynomial approximation. For notational simplicity, we denote the spatial coordinate of the curve as $\chi\left(u_{m}\right), \chi \in\left\{x_{0}, y_{0}, z_{0}\right\}$; our objective is to determine the derivative of the curve $\chi_{u}\left(u_{m}\right)$.

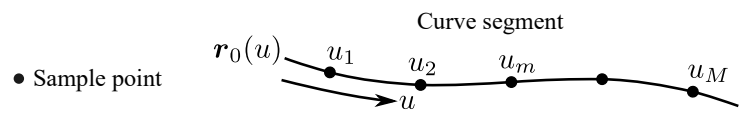

FIGURE 5. Discrete curve segment having $M$ sample points.

\section{1) Finite Difference Approximation}

The simplest solution to numerically evaluate the derivative $\chi_{u}\left(u_{m}\right)$ is use of the finite difference approximation. If the central difference is employed, numerical differentiation is given as follows:

$$
\chi_{u}\left(u_{m}\right) \approx \frac{\chi\left(u_{m+1}\right)-\chi\left(u_{m-1}\right)}{u_{m+1}-u_{m-1}} .
$$

Note that the forward sample at $u_{m+1}$ or backward sample $u_{m-1}$ is unavailable, we can utilize the backward or the forward difference instead of the central difference. Eq. (37) can be used to obtain $x_{0 u}$ and $y_{0 u}$, and then, the coefficient $\alpha_{u}$ defined in Eq. (22a) and the corresponding correction factor given in Eq. (26) can be evaluated numerically.

The advantage of the finite difference approximation is its computational simplicity. If the scanning curve is a smooth curve such as an ellipse or a polynomial curve discussed in Section II-D, use of the finite difference approximation is a reasonable option. However, when the scanning curve strongly fluctuates because the antenna is mounted on an unstable platform such as an aircraft or a ground vehicle, the finite difference approximation is possibly numerically unstable. In such a case, the polynomial approximation descried next is an useful solution.

\section{2) Polynomial Approximation}

In Section II-D2, we derived the correction factor for a polynomial scanning curve with known polynomial coefficients. We can extend this correction factor to a discrete scanning curve; that is, we apply polynomial fitting to the discrete curve segment shown in Fig. 5. In this case, polynomial coefficients are unknown and they must be determined from the discrete locations of the scanning curve $\chi\left(u_{m}\right)$.

As was done in Eq. (33), we consider the following expansion of the discrete curve at the sample point $u_{m}$ :

$$
\begin{aligned}
\chi\left(u_{m}\right) & =\sum_{p=0}^{K} a_{p}^{(\chi)} u_{m}^{p}=\boldsymbol{u}_{m}^{T} \boldsymbol{a}^{(\chi)} \\
\boldsymbol{u}_{m} & =\left[1, u_{m}, u_{m}^{2}, \ldots, u_{m}^{p}, \ldots, u_{m}^{K}\right]^{T} \\
\boldsymbol{a}^{(\chi)} & =\left[a_{0}^{(\chi)}, a_{1}^{(\chi)}, \cdots, a_{p}^{(\chi)}, \cdots, a_{K}^{(\chi)}\right]^{T}
\end{aligned}
$$

where $[\cdot]^{T}$ is the transpose operation, $a_{p}^{(\chi)}$ is the unknown $p$ th order expansion coefficient to be determined, $[\cdot]^{(\chi)}$ designates that this coefficient is related to the coordinate $\chi \in$ $\left\{x_{0}, y_{0}, z_{0}\right\}$, and $K$ is the maximum order of the polynomial. 
Considering Eq. (38) at all sample points within the discrete curve segment results in the following expression:

$$
\begin{aligned}
\boldsymbol{\chi} & =\boldsymbol{U} \boldsymbol{a}^{(\chi)} \\
\chi & =\left[\chi\left(u_{1}\right), \chi\left(u_{2}\right), \ldots, \chi\left(u_{m}\right), \ldots, \chi\left(u_{M}\right)\right]^{T} \\
\boldsymbol{U} & =\left[\boldsymbol{u}_{1}, \boldsymbol{u}_{2}, \ldots, \boldsymbol{u}_{m}, \ldots, \boldsymbol{u}_{M}\right]^{T}
\end{aligned}
$$

The least squares solution to the coefficient vector $\boldsymbol{a}^{(\chi)}$ is given by

$$
\boldsymbol{a}^{(\chi)}=\left(\boldsymbol{U}^{T} \boldsymbol{U}\right)^{-1} \boldsymbol{U}^{T} \boldsymbol{\chi}
$$

Using the coefficient vector $\boldsymbol{a}^{(\chi)}$, the discrete curve can be represented within the curve segment of Fig. 5 as

$$
\begin{aligned}
& \chi(u)=\boldsymbol{u}^{T}(u) \boldsymbol{a}^{(\chi)} \\
& \boldsymbol{u}(u)=\left[1, u, u^{2}, \ldots, u^{m}, \ldots, u^{K}\right]^{T} .
\end{aligned}
$$

Therefore, we can readily evaluate the derivative $\chi_{u}(u)$ via

$$
\begin{aligned}
\chi_{u}(u) & \approx \boldsymbol{u}^{\prime T}(u) \boldsymbol{a}^{(\chi)} \\
\boldsymbol{u}^{\prime}(u) & =\left[0,1,2 u, \ldots, m u^{(m-1)}, \ldots, K u^{(K-1)}\right]^{T} .
\end{aligned}
$$

We repeat the aforementioned procedure for $\chi \in\left\{x_{0}, y_{0}\right\}$ to obtain the derivatives $x_{0 u}$ and $y_{0 u}$ numerically, and then, we can evaluate the coefficient $\alpha_{u}$ defined in Eq. (22a) and the corresponding correction factor given in Eq. (26). A more complicated case of 2-D antenna scanning along an arbitrary curved surface is treated in [13].

\section{F. RADAR CROSS-SECTION COMPUTATION}

The final step of the image-based RCS measurement is to compute RCS from the reconstructed 2-D spatial image $\psi(\boldsymbol{r})$. Basically, this can be accomplished by taking the 2$\mathrm{D}$ inverse Fourier transform of the reconstructed 2-D image $\psi(\boldsymbol{r})$ with respect to the spatial variables $x$ and $y$. The following derivation is similar to those presented in [8], and an extension to 3-D imaging is discussed in [13].

Because we can consider the reconstructed 2-D image $\psi(\boldsymbol{r})$ as a cluster of point scatterers, we can compute the scattered field at an arbitrary location $\boldsymbol{r}_{0}$ created by the 2-D reflectivity distribution according to Eq. (5), as follows:

$$
E^{s}\left(k, \boldsymbol{r}_{0}\right)=\frac{k^{2}}{\sqrt{4 \pi}} \int_{-\infty}^{\infty} \int_{-\infty}^{\infty} \psi(\boldsymbol{r}) \frac{e^{-2 j k\left|\boldsymbol{r}_{0}-\boldsymbol{r}\right|}}{\left|\boldsymbol{r}_{0}-\boldsymbol{r}\right|^{2}} d x d y
$$

In Eq. (43), the following expansion can be made [19]:

$$
\begin{aligned}
\left|\boldsymbol{r}_{0}-\boldsymbol{r}\right| & =\sqrt{\left(\boldsymbol{r}_{0}-\boldsymbol{r}\right) \cdot\left(\boldsymbol{r}_{0}-\boldsymbol{r}\right)} \\
& =\sqrt{\left|\boldsymbol{r}_{0}\right|^{2}-2 \boldsymbol{r}_{0} \cdot \boldsymbol{r}+|\boldsymbol{r}|^{2}} \\
& =\left|\boldsymbol{r}_{0}\right|\left[1-\frac{\widehat{\boldsymbol{r}}_{0} \cdot \boldsymbol{r}}{\left|\boldsymbol{r}_{0}\right|}+O\left(\frac{|\boldsymbol{r}|^{2}}{\left|\boldsymbol{r}_{0}\right|^{2}}\right)\right] \\
& =\left|\boldsymbol{r}_{0}\right|-\widehat{\boldsymbol{r}}_{0} \cdot \boldsymbol{r}+O\left(|\boldsymbol{r}|^{2} /\left|\boldsymbol{r}_{0}\right|\right) \\
\widehat{\boldsymbol{r}}_{0} & =\boldsymbol{r}_{0} /\left|\boldsymbol{r}_{0}\right|=\boldsymbol{r}_{0} / r_{0}
\end{aligned}
$$

Using (44), we obtain the following far-field approximation:

$$
\begin{aligned}
1 /\left|\boldsymbol{r}_{0}-\boldsymbol{r}\right|^{2} & \approx 1 / r_{0}^{2} \\
e^{-2 j k\left|\boldsymbol{r}_{0}-\boldsymbol{r}\right|} & \approx e^{-j 2 k r_{0}} e^{j 2 k \widehat{\boldsymbol{r}}_{0} \cdot \boldsymbol{r}}=e^{-j 2 k r_{0}} e^{j \boldsymbol{k}_{r} \cdot \boldsymbol{r}} \\
\boldsymbol{k}_{r} & =2 k \widehat{\boldsymbol{r}}_{0} .
\end{aligned}
$$

Substituting Eq. (45) into Eq. (43) yields in the following approximation of the scattered field:

$$
\begin{aligned}
E^{s}\left(k, \boldsymbol{r}_{0}\right) & \approx \frac{k^{2} e^{-j 2 k r_{0}}}{r_{0}^{2} \sqrt{4 \pi}} \int_{-\infty}^{\infty} \int_{-\infty}^{\infty} \psi(\boldsymbol{r}) \exp \left(j \boldsymbol{k}_{r} \cdot \boldsymbol{r}\right) d x d y \\
& =\frac{k^{2} e^{-j 2 k r_{0}}}{r_{0}^{2} \sqrt{4 \pi}} \mathcal{F}_{(x, y)}^{-1}[\psi(\boldsymbol{r})]
\end{aligned}
$$

where $\mathcal{F}_{(x, y)}^{-1}[\cdot]$ is the inverse Fourier transform with respect to the spatial variables $(x, y)$.

The definition of RCS is

$$
\sigma\left(\boldsymbol{k}_{r}\right)=\lim _{r_{0} \rightarrow \infty} 4 \pi r_{0}^{2}\left|\frac{E^{s}\left(k, \boldsymbol{r}_{0}\right)}{E_{0}^{i}\left(k, \boldsymbol{r}_{0}\right)}\right|^{2}
$$

where $E_{0}^{i}\left(k, \boldsymbol{r}_{0}\right)$ is the incident field given by

$$
E_{0}^{i}\left(k, \boldsymbol{r}_{0}\right)=\frac{e^{-j k r_{0}}}{r_{0}} .
$$

Therefore, by combining Eqs. (46), (48), and (47), we finally obtain the following expression:

$$
\sigma\left(\boldsymbol{k}_{r}\right)=k^{4}\left|\mathcal{F}_{(x, y)}^{-1}[\psi(\boldsymbol{r})]\right|^{2}
$$

To obtain the RCS as a function of the azimuthal angle with respect to a certain location on the image plane, the following procedure must be taken:

1) Specify the desired (angular) frequency within the frequency band used for the scattering experiment and the corresponding wavenumber $k=\omega / c$.

2) Select the reference point within the image plane, denoting $\boldsymbol{r}_{r}$. This reference point should be as close as possible from all scatterers within the image plane. If the scatterers are distributed around the $z$-axis, the appropriate reference point is $\boldsymbol{r}_{r}=\left(0,0, z_{c}\right)$. If the scatterers are around a off-centered location, the reference point should be set at that point.

3) Specify the desired local azimuthal angle $\alpha$ for the reference point, and determine the corresponding antenna location $\boldsymbol{r}_{0}$ on the scanning curve. This is possible as we have assumed that every points on the scanning curve can be uniquely determined from the azimuthal angle $\alpha$.

4) Using the determined antenna location $\boldsymbol{r}_{0}$, compute local zenith angle $\beta$ based on Eq. (15b).

5) Construct the wavenumber vector $\boldsymbol{k}_{r}=\left(k_{x}, k_{y}, k_{z}\right)$ based on Eq. (18).

6) Calculate the RCS according to Eq. (49).

Finally, we require calibrating the obtained RCS by using a calibration target with a known $\operatorname{RCS} \sigma_{c 0}\left(\alpha_{c}\right)$ at the azimuthal angle $\alpha_{c}$. Assuming that we have two computed data from Eq. (49), denoting $\sigma_{t}\left(\alpha_{c}\right)$ and $\sigma_{c}\left(\alpha_{c}\right)$, where the former and 
the latter are RCS of the target and the calibration target, respectively, we can accomplish the calibration as follows:

$$
\widehat{\sigma}(\alpha)=\sigma_{t}(\alpha) \cdot \sigma_{c 0}\left(\alpha_{c}\right) / \sigma_{c}\left(\alpha_{c}\right)
$$

The Eq. (50) completes the overall process of the RCS determination based on synthetic aperture imaging.

Note that although one can place a desired target only when the measurement is carried out in an anechoic chamber as explained in Section IV, this is not the case for airborne SAR observation as presented in Section V. For the latter situation, we can apply a spatial filter on the reconstructed image $\psi(\boldsymbol{r})$ to isolate a target of interest [18], [20]. For example, we consider the following 2-D spatial window function represented by a disk of unity radius:

$$
w(r)= \begin{cases}1, & |r| \leq 1 \\ 0, & \text { otherwise }\end{cases}
$$

To isolate an interested disk-shaped area of radius $a_{w}$ centered at the reference point $\boldsymbol{r}_{r}$, the following spatial window is applied:

$$
\widehat{\psi}(\boldsymbol{r})=\psi(\boldsymbol{r}) w\left(\left|\boldsymbol{r}-\boldsymbol{r}_{r}\right| / a_{w}\right) .
$$

Then, RCS of the target within this area can be computed by Eq. (49) with $\psi(\boldsymbol{r}) \rightarrow \widehat{\psi}(\boldsymbol{r})$.

\section{G. SUMMARY OF THE PROPOSED ALGORITHM}

The proposed image-based RCS determination algorithm is summarized as follows:

1) Specify the altitude $z_{c}$ of the $(x, y)$-plane in which 2-D image is reconstructed. We refer to this $(x, y)$-plane at the altitude $z_{c}$ as the image plane.

2) For each image pixel at $(x, y)$ within the selected image plane, compute the antenna position $\left(x_{0}^{\prime}, y_{0}^{\prime}, z_{0}^{\prime}\right)$ in the local coordinate centered at the pixel based on Eq. (2).

3) Compute the derivative $\alpha_{u}$ using Eq. (15c) and Eq. (22a) to obtain the correction factor defined in Eq. (24). The differential coefficients $x_{0 u}$ and $y_{0 u}$ and the corresponding correction factor can be exactly evaluated if the antenna scanning curve is represented by an analytical expression such as those exemplified in Section II-D. If such an analytical representation is unavailable, one can use the numerical correction factor described in Section II-E.

4) Based on the calculated correction factor $g\left(k, \boldsymbol{r}_{0}, \boldsymbol{r}\right)$, reconstruct 2-D spatial image $\psi(\boldsymbol{r})$ within the image plane $\left(x, y, z_{c}\right)$ based on Eq. (10) or its discretized version of Eq. (12).

5) Repeat steps 2-4 for all pixels with in the image plane $\left(x, y, z_{c}\right)$.

6) Compute RCS via the inverse Fourier transformation of the reconstructed 2-D image $\psi(\boldsymbol{r})$ with respect to the spatial variables $(x, y)$. For this computation, an appropriate reference point $\boldsymbol{r}_{r}$ must be selected as explained in Section II-F

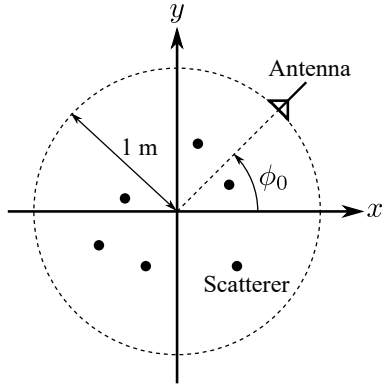

(a)

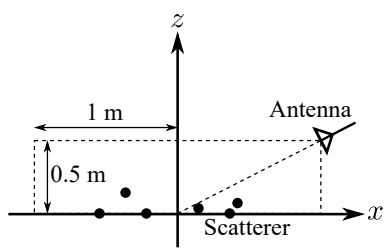

(b)
FIGURE 6. Common geometry of the simulations. Small spherical scatterers were distributed, where some scatterers had nonzero altitude. Image plane $(x, y)$ was selected to $z_{c}=0$. (a) Top view. (b) Side view.

7) Steps 1-5 are applied for the received data of a target under test and a calibration target to derive uncalibrated RCS for each target. Then, the RCS is calibrated using Eq. (50).

\section{NUMERICAL SIMULATION}

In this section, the proposed algorithm is validated by numerical simulations based on the discrete scatterer model given in Eq. (8). We investigate the effectiveness of the derived correction factor and its applicability to an arbitrarily curved scanning trajectory. Moreover, effects of a scatterer with nonzero altitude and an off-centered scatterer are considered in detail.

\section{A. OVERVIEW OF THE SIMULATION}

Fig. 6 illustrates the common geometry used in the following series of numerical simulations, and Table 1 lists the corresponding simulation parameters. We considered a complete circular trajectory having the radius and the altitude of $1 \mathrm{~m}$ and $0.5 \mathrm{~m}$, respectively. Small spherical scatterers were distributed in the 3-D space, where some of them have nonzero altitude from the image plane $(x, y)$ at $z_{c}=0$. The center frequency and the bandwidth were selected to $10 \mathrm{GHz}$ and $4 \mathrm{GHz}$, respectively. The transmitting and the receiving antennas were collocated and assumed to have an isotropic antenna pattern; the antennas were scanned in steps of $0.5^{\circ}$. Although we could exactly determine the correction factor for this circular trajectory using Eq. (30), we used the numerical correction factor discussed in Section II-E throughout the series of simulations. In this computation, the number of points in a curve segment was $M=11$, and the order of the polynomial was $K=2$.

For each simulation result, we compared the RCS derived from the SAR image with the theoretical RCS of a cloud of point scatterers given by

$$
\sigma=k^{4}\left|\sum_{i} C_{i} \exp \left(j k_{x} x_{i}+j k_{y} y+j k_{z} z\right)\right|^{2}
$$

where $C_{i}$ is given by Eq. (7) for a small conducting sphere, and the spatial frequencies $\left(k_{x}, k_{y}, k_{z}\right)$ for the azimuthal and 
TABLE 1. Simulation parameters.

\begin{tabular}{l|l}
\hline Center frequency & $10 \mathrm{GHz}$ \\
Bandwidth & $4 \mathrm{GHz}$ \\
Frequency step & $10 \mathrm{MHz}$ \\
Antenna scanning curve & Circle \\
Angular interval & {$\left[-180^{\circ}, 180^{\circ}\right]$} \\
Angular step & $0.5^{\circ}$ \\
Antenna altitude & $0.5 \mathrm{~m}$ \\
Radius of the scanning circle & $1.0 \mathrm{~m}$ \\
Number of points in a curve segment & $M=11$ \\
Order of the polynomial & $K=2$ \\
Scatterer & $\mathrm{Small} \mathrm{spheres}$ \\
Pixel spacing & $4 \mathrm{~mm}$ \\
\hline
\end{tabular}

zenith angle $(\alpha, \beta)$ are given by Eq. (18).

\section{B. IMPROVEMENT BY THE CORRECTION FACTOR}

In this first simulation, our objective was to show the improvement of the RCS computation by introducing the proposed correction factor. In addition to the regular circular path, we considered a modulated circular trajectory expressed by the following equation to show the applicability of the proposed correction factor to an arbitrarily curved scanning path:

$$
\begin{aligned}
x_{0}(u) & =\left[a+a_{\Delta}(u)\right] \cos u \\
y_{0}(u) & =\left[a+a_{\Delta}(u)\right] \sin u \\
a_{\Delta}(u) & =a^{\prime} \cos (\xi u)
\end{aligned}
$$

where we selected the amplitude $a^{\prime}$ and the frequency $\xi$ to $5 \mathrm{~cm}$ and 10, respectively. Although one may derive the exact correction factor for the curve given in Eq. (54), we used numerical correction factor described in Section II-E.

Fig. 7(a) and (b) show the geometry of the simulation and the reconstructed spatial image using the regular circular path, respectively. The altitudes of these scanning curve was at $z_{0}=0.5 \mathrm{~m}$, as illustrated in Fig. 6. As depicted in Fig. 7(a), three spherical scatterers were placed at $\boldsymbol{r}_{1}=\left(x_{1}, y_{1}, z_{1}\right)=$ $(-30,30,0) \mathrm{cm}, \boldsymbol{r}_{2}=\left(x_{2}, y_{2}, z_{2}\right)=(-20,30,0) \mathrm{cm}$, and $\boldsymbol{r}_{3}=\left(x_{3}, y_{3}, z_{3}\right)=(-10,30,0) \mathrm{cm}$, where the radii of them were $a_{1}=0.5 \mathrm{~mm}, a_{2}=1.0 \mathrm{~mm}$, and $a_{3}=1.5 \mathrm{~mm}$ $\left(k a_{i}<0.4\right)$, respectively. Note that these scatterers were intentionally off-centered to clearly show the improvement by the correction factor. The spatial image of Fig. 7(b) shows the responses of these three spherical scatterer, where a larger radius corresponds to a brighter response.

Figs. 8 and 9 show the calculated RCS for the circular and the modulated circular trajectories, respectively, plotted with the theoretical RCS defined in Eq. (53). In these figures, (a) and (b) show the RCS without (i.e., $g\left(k, \boldsymbol{r}_{0}, \boldsymbol{r}\right)=1$ ) and with the correction factor, respectively. In computing RCS, we assumed the reference point to be $\boldsymbol{r}_{2}$. Both Figs. 8(b) and 9(b) show the improvement by the proposed correction factor as compared to Figs. 8(b) and 9(b). Therefore, we confirm the feasibility of the proposed numerical correction factor for an arbitrary scanning curve.

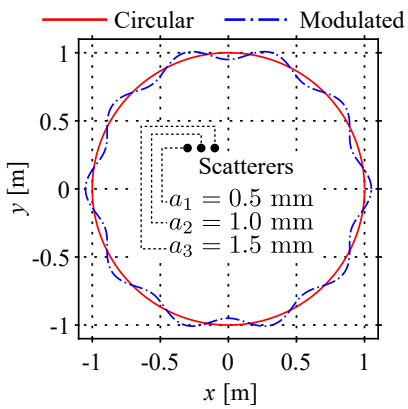

(a) (b)

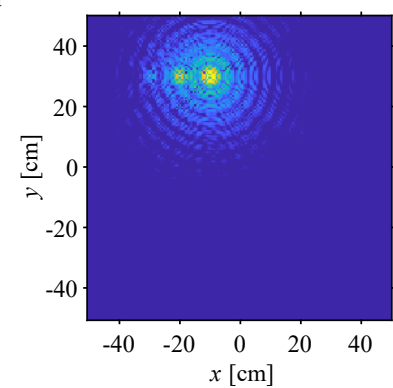

FIGURE 7. Geometry of the simulation and the reconstructed spatial image. Three small spherical scatterers having different radii were placed at the off-centered locations. In addition to the regular circular trajectory, modulated circular path was tested. (a) Geometry. (b) Spatial image of the circular path.

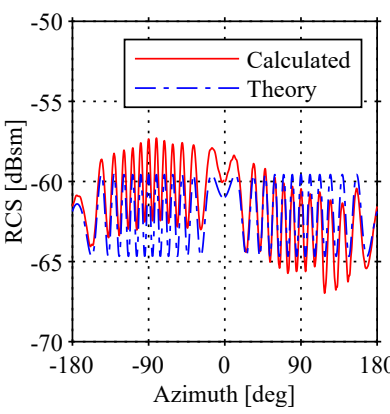

(a)

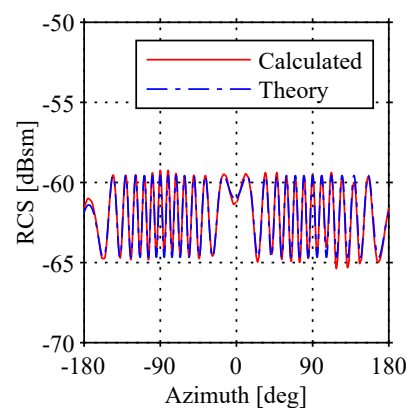

(b)
FIGURE 8. RCS derived from SAR image with the regular circular trajectory. (a) Without the correction factor. (b) With the correction factor.

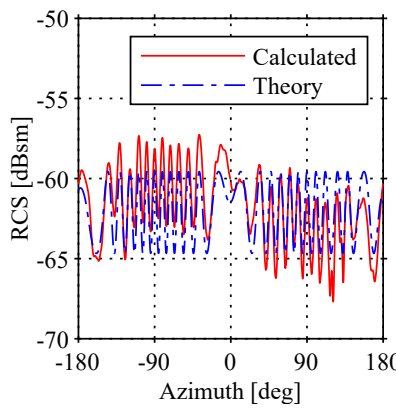

(a)

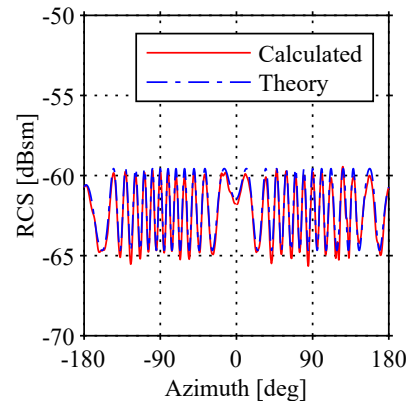

(b)
FIGURE 9. RCS derived from SAR image with the modulated circular trajectory. (a) Without the correction factor. (b) With the correction factor.

\section{EFFECTS OF A SCATTERER WITH NONZERO ALTITUDE}

The second simulation was given to investigate the effects of a scatterer with nonzero altitude. Before discussing the simulation result, let us consider the situation shown in Fig. 10 as an example; two point scatterers are located within the $(x, z)$-plane at $y=0$, where the altitude of the scatterer 


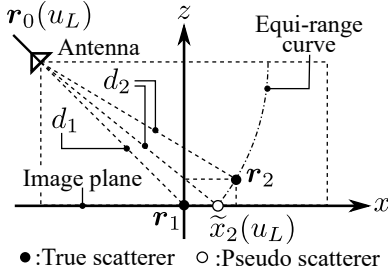

(a)

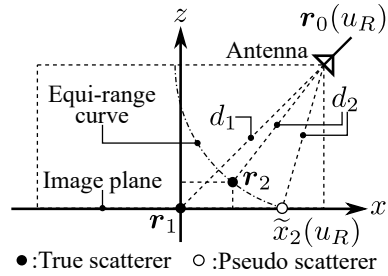

(b)
FIGURE 10. Example geometry of scatterer with nonzero altitude. (a) Antenna on the left side. (b) Antenna on the right side.

at $\boldsymbol{r}_{1}=\left(x_{1}, y_{1}, z_{1}\right)$ is zero $\left(z_{1}=0\right)$ and the scatterer at $\boldsymbol{r}_{2}=\left(x_{2}, y_{2}, z_{2}\right)$ has nonzero altitude $\left(z_{2} \neq 0\right)$. In Figs. 10(a) and (b), the antenna is on the left (negative) and right (positive) side of the $x$-axis, representing the antenna locations by $\boldsymbol{r}_{0}\left(u_{L}\right)$ and $\boldsymbol{r}_{0}\left(u_{R}\right)$, respectively. We denote distance from the antenna at $\boldsymbol{r}_{0}(u)$ to the scatterers at $\boldsymbol{r}_{1}$ and $\boldsymbol{r}_{2}$ as $d_{1}(u)$ and $d_{2}(u)$, respectively. As mentioned earlier, the image plane is assumed to be at $z_{c}=0$.

First, we consider the case shown in Fig. 10(a). Because the scatterer at $\boldsymbol{r}_{1}$ is within the image plane, this scatterer is reconstructed at the exactly correct location in the image. However, as for the scatterer at $\boldsymbol{r}_{2}$, it is projected onto the image plane such that the $x$-position of this scatterer, represented by $\widetilde{x}_{2}\left(u_{L}\right)$, is displaced toward $\boldsymbol{r}_{1}$ such that $\widetilde{x}_{2}\left(u_{L}\right)<x_{2}$; this effect is well-known as layover in airborne or spaceborne SAR images of steep topography. As illustrated in Fig. 10(a), the projected location $\widetilde{x}_{2}\left(u_{L}\right)$ is the point at which the $x$-axis and the equi-range curve for the scatterer at $\boldsymbol{r}_{2}$, that is, a circle of radius $d_{2}\left(u_{L}\right)$ centered at $\boldsymbol{r}_{0}\left(u_{L}\right)$, intersects each other. We refer to the projected scatterer as "pseudo scatterer" in the following discussion. Next, let us focus on the case shown in Fig. 10(b). By the similar discussion as the previous case of Fig. 10(a), the scatterer is projected onto the image plane, appearing as a pseudo scatter on the $x$-axis. In this case, the $x$-position of the pseudo scatterer $\widetilde{x}_{2}\left(u_{R}\right)$ is displaced away from the true location $x_{2}$ so that $\widetilde{x}_{2}\left(u_{L}\right)>x_{2}$.

As can be understood from this example, the location of the pseudo scatterer strongly depends on the antenna location $\boldsymbol{r}_{0}(u)$ when the scatterer has nonzero altitude from the image plane. Because we are trying to reconstruct RCS from a 2-D image, the predicted RCS is those for the pseudo scatterers on the 2-D image plane rather than true scatterers distributed over 3-D spatial domain. Let us take a closer look at this effect by using a numerical example shown in Fig. 11, where both spherical scatterers having the same radii $a_{i}=1 \mathrm{~mm}$ were on the $x$-axis in Fig. 11(a), and one of the scatterers had an altitude of $20 \mathrm{~cm}$ in Fig. 11(b). The spatial images reconstructed at $z_{c}=0$ corresponding to the former and the latter cases are shown in Figs. 12(a) and (b), respectively. Because both the scatterers were on the image plane in Fig. 11(a), the reconstructed image shows two responses for these scatterers at exactly true locations. However, as for the scatterers at $\boldsymbol{r}_{2}$ in Fig. 11(b) which had nonzero altitude, the

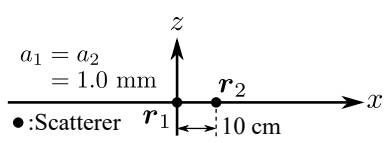

(a)

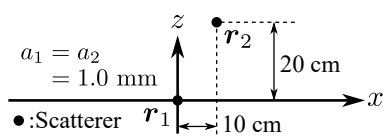

(b)
FIGURE 11. Geometry of the simulation for demonstrating the effect of the scatterer with nonzero altitude. (a) Both scatterers were on the $x$-axis. (b) Scatterer on the right has nonzero altitude $\left(z_{2}=20 \mathrm{~cm}\right)$.

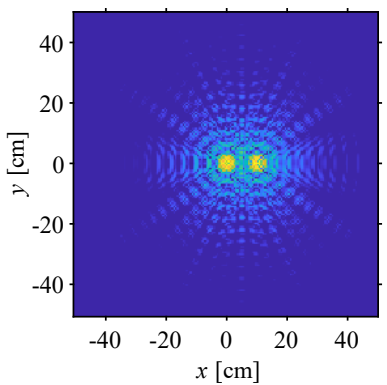

(a)

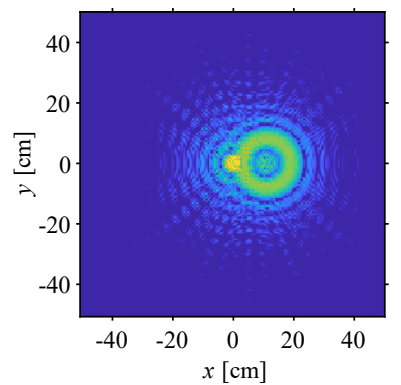

(b)
FIGURE 12. Reconstructed spatial image of spherical scatterers having different altitudes. (a) Both scatterers were on the $x$-axis. (b) Scatterer on the right has nonzero altitude $\left(z_{2}=20 \mathrm{~cm}\right)$.

reconstructed image shows ring-shaped distribution around the scatterer at on the right side. This ring-shaped response is what we have explained as the pseudo scatterer in Fig. 10.

To see how this pseudo scatterer affects the RCS prediction, we transformed the spatial images into the spatial frequency domain via the 2-D inverse Fourier transform of the images shown in Fig. 12 with respect to $x$ and $y$. The result is displayed in Fig. 13, where (a) and (b) are the spatial frequency spectra correspond to Figs. 12(a) and (b), respectively. These spectra show the interference pattern of the two point scatterers; in Fig. 13(a), one can recognize vertical stripes on the annulus-like spectrum. This was because that the two point scatterers were separated horizontally, and the distance between the two point scatterers on the image plane was unchanged with respect to the antenna location; as two scatterers have equal $y$-coordinates $\left(y_{1}=y_{2}=0\right)$, no interference pattern was observed along the $k_{x}$-direction as shown in Fig. 13(a).

However, the situation was quite different for the case of the scatterer with nonzero altitude shown in Fig. 10(b) and the corresponding spatial frequency spectrum of Fig. 13(b); the interference pattern shown in Fig. 13(b) is no longer vertically straight stripes, in contrast to Fig. 13(a). This can be described by the pseudo scatterer illustrated in Fig. 10. Let us focus on the period of the interference pattern along $k_{x}$-axis in Fig. 13(b). If the antenna was located at $\boldsymbol{r}_{0}\left(u_{L}\right)$ in Fig. 10(a), the pseudo scatterer at $\widetilde{x}_{2}\left(u_{L}\right)$ was displaced toward the another scatterer at $x_{1}=0$ compared to the actual location $x_{2}=10 \mathrm{~cm}$, that is, $\widetilde{x}_{2}<x_{2}$. Therefore, the period of the interference pattern along the negative $k_{x}$-axis was lower than that of the zero-altitude scatterer shown in Fig. 


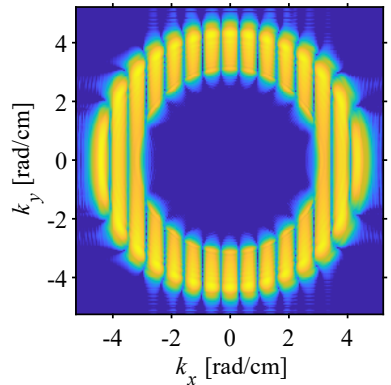

(a)

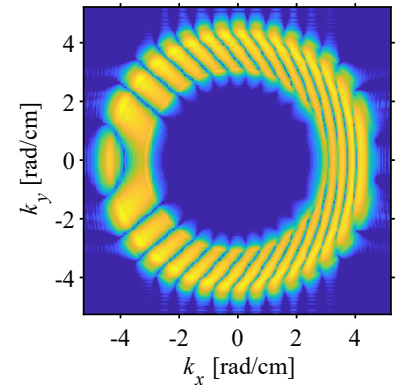

(b)
FIGURE 13. Spatial frequency spectra for spherical scatterers having different altitudes. (a) Both scatterers were on the $x$-axis. (b) Scatterer on the right has nonzero altitude $\left(z_{2}=20 \mathrm{~cm}\right)$.

13(a). In the case shown in Fig. 10(b), as the pseudo scatter at $\widetilde{x}_{2}$ was displaced away from the scatterer at $x_{1}$ compared to the original location $x_{2}$ such that $\widetilde{x}_{2}>x_{2}$, the period of the interference pattern along the positive $k_{x}$-axis was higher than that of Fig. 13(a).

The effect of the pseudo scatterer was also apparent in the calculated RCS. Figs. 14(a) and (b) show the RCS derived from the spatial frequency spectra shown in Figs. 13(a) and (b), respectively, where the reference point was set at the origin such that $\boldsymbol{r}_{r}=\boldsymbol{r}_{1}=(0,0,0)$. The calculated and the theoretical RCS excellently agreed with each other as shown in Fig. 14(a), because the two scatterers were on the image plane. However, the RCS shown in Fig. 14(b) obviously differs from the theoretical value, because of the deformed interference pattern shown in Fig. 13(b) owing to the nonzero altitude of the scatterer. In conclusion, the altitude of the image plane should be selected such that the displacement of the scatterers from the image plane be as small as possible. As can be understood from Fig. 10, the displacement of the pseudo scatterer from the actual location becomes greater as the zenith angle $\beta$ at the scatterer increases. If this effect cannot be ignored, full 3-D synthetic aperture imaging with 2-D surface antenna scanning [11]-[13] is required to achieve precise RCS measurement.

\section{EFFECTS OF AN OFF-CENTERED SCATTERER}

In the last simulation, we deal with effect of an off-centered scatter. We consider the geometry illustrated in Fig. 15 as an example, where two point scatterers were placed on the $x$-axis; one is at the origin $\left(\boldsymbol{r}_{1}\right)$ and another is at an offcentered location on the positive side of the $x$-axis $\left(\boldsymbol{r}_{2}\right)$. As in the Section III-C, the antenna is on the left side location, denoting $\boldsymbol{r}_{0}\left(u_{L}\right)$, in Fig. 15(a) and on the right side location, representing $\boldsymbol{r}_{0}\left(u_{R}\right)$, in Fig. 15(b). For these scatterers, we consider how local zenith angle at the scatterer, denoted by $\beta_{i}(u)$ for the antenna at $\boldsymbol{r}_{0}(u)$, varies with respect to the antenna location on the circular path. For the scatterer at $\boldsymbol{r}_{1}$, the zenith angle $\beta_{1}(u)$ is unchanged with respect to the antenna location since this scatterer is at the origin. However,

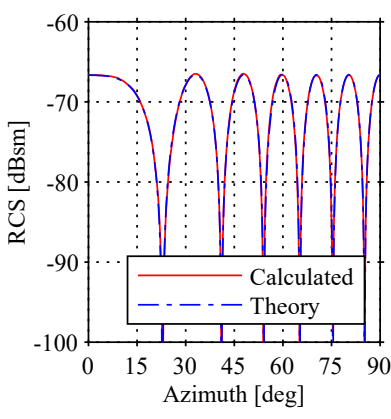

(a)

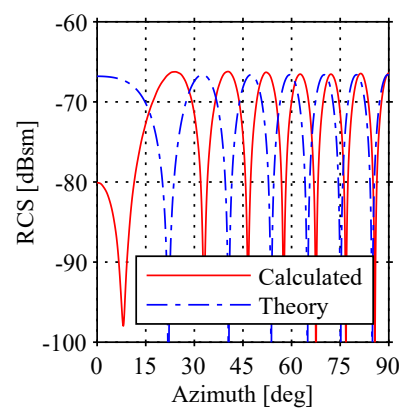

(b)
FIGURE 14. RCS of spherical scatterers having different altitudes. (a) Both scatterers were on the $x$-axis. (b) Scatterer on the right has nonzero altitude $\left(z_{2}=20 \mathrm{~cm}\right)$.

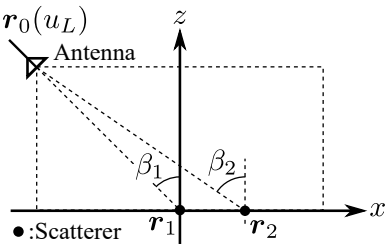

(a)

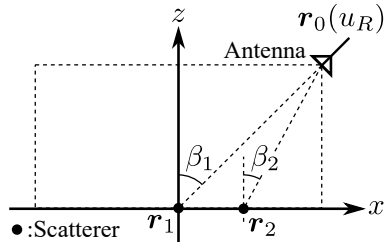

(b)
FIGURE 15. Example geometry of the off-centered scatterer. (a) Antenna on the left side. (b) Antenna on the right side.

as for the off-centered scatterer at $\boldsymbol{r}_{2}$, the zenith angle $\beta_{2}(u)$ depends on the antenna location, where $\beta_{2}\left(u_{L}\right)>\beta_{2}\left(u_{R}\right)$ in this example. Moreover, the zenith angles at each scatterer, $\beta_{1}(u)$ and $\beta_{2}(u)$, generally differ from each other. Therefore, unless the antenna scanning trajectory is on the image plane $\left(z_{0}=0\right)$, the local zenith angle $\beta_{i}(u)$ varies differently for each scatterer. This means that the reconstructed 2-D image contains the information about the RCS of the scatterer observed from various zenith angles.

To see how this angular difference affects to the RCS computation, let us consider the spatial frequency domain representation of the point scatterers. Figs. 16 and 17 show the 3-D spatial frequency support corresponding to the scatterers at $\boldsymbol{r}_{1}$ and $\boldsymbol{r}_{2}$ shown in Fig. 15, respectively, where (a) and (b) show the support projected onto the $\left(k_{x}, k_{y}\right)$-plane and the $\left(k_{x}, k_{z}\right)$-plane, respectively. Note that the support was determined by Eq. (18) as a function of the azimuthal angle of the antenna $u$ and the wavenumber $k$ within the frequency band given in Table 1, where the local azimuthal and zenith angle $(\alpha, \beta)$ is determined from Eqs. (15a) and (15b). Although the spatial frequency support has the 3-D structure unless the antenna and the scatterers are within the image plane, what we can obtain from the 2-D spatial image is the projected 2-D support on the $\left(k_{x}, k_{y}\right)$-plane as shown in Figs. 16(a) and 17(a).

As shown in Fig. 16, the spatial frequency support of the scatterer at $\boldsymbol{r}_{1}$ has a regular conical structure of which the cone angles measured from the $k_{z}$-axis are $\beta\left(u_{R}\right)=\beta\left(u_{L}\right)$ 


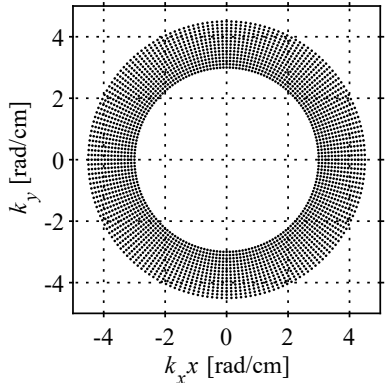

(a)

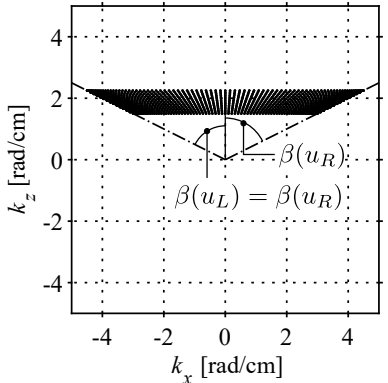

(b)
FIGURE 16. Spatial frequency support for the scatterer at the origin. (a) $\left(k_{x}, k_{y}\right)$-plane. (b) $\left(k_{x}, k_{z}\right)$-plane.

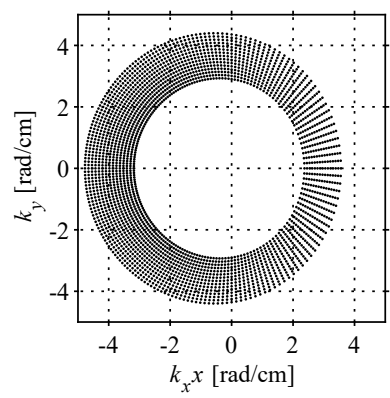

(a)

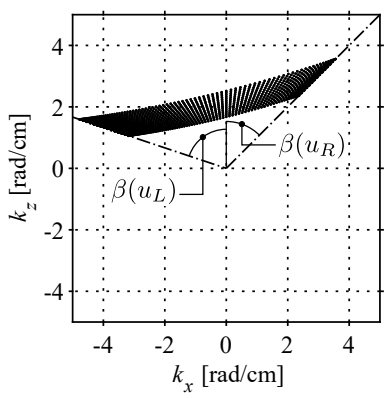

(b)
FIGURE 17. Spatial frequency support for the off-centered scatterer. (a) $\left(k_{x}, k_{y}\right)$-plane. (b) $\left(k_{x}, k_{z}\right)$-plane.

that are unchanged with respect to the antenna location. This means that the RCS extracted from the 2-D spatial frequency spectrum shown in Fig. 16(a) corresponds to the RCS viewed from the constant zenith angle $\beta$ for all the local azimuthal angle $\alpha$. However, as shown in Fig. 17, the spatial frequency support of the off-centered scatterer at $\boldsymbol{r}_{2}$ has a conical structure tilted toward the negative $k_{x^{-}}$direction, where the cone angles measured from the $k_{z}$-axis are $\beta\left(u_{L}\right)$ and $\beta\left(u_{R}\right) \neq \beta\left(u_{L}\right)$ for the antenna location at $\boldsymbol{r}_{0}\left(u_{L}\right)$ and $\boldsymbol{r}_{0}\left(u_{R}\right)$, respectively. This means that, in contrast to the centered scatterer at $\boldsymbol{r}_{1}$, the corresponding zenith angle $\beta(u)$ of the resultant RCS derived from the 2D spatial frequency spectrum shown in Fig. 17(a) varies with respect to the antenna location $\boldsymbol{r}_{0}(u)$. Therefore, if this offcentered scatterer has a directional RCS pattern in the zenith direction, the derived azimuthal RCS shows directivity even if this scatterer has an omni-directional RCS pattern in the azimuthal direction.

To demonstrate the aforementioned phenomenon, we consider the numerical examples for which the location of the scatterer is shown in Fig. 18, where (a) and (b) are the case for a centered and an off-centered scatterer, respectively. In this simulation, two spherical point scatterers of radii $1 \mathrm{~mm}$ were vertically stacked such that the $(x, y)$-coordinate coincides

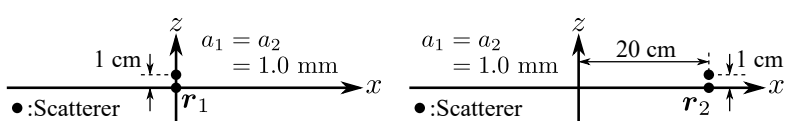

(a)

(b)

FIGURE 18. Geometry of the simulation for demonstrating the effect of the off-centered scatterer. (a) Scatterers on the origin. (b) Scatterers on the off-centered location.

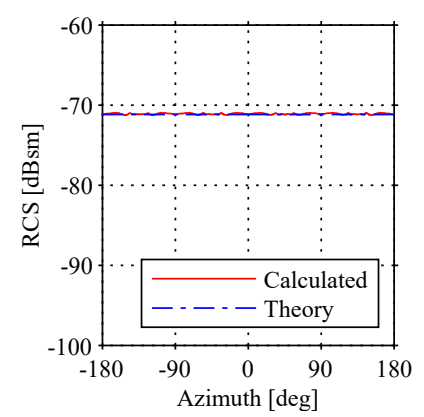

(a)

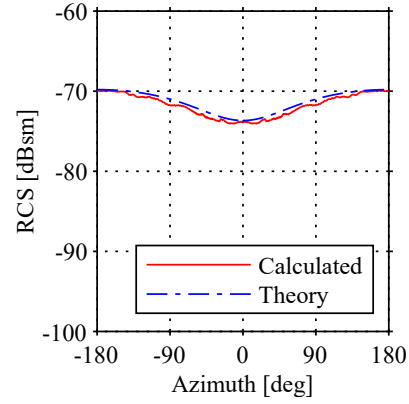

(b)
FIGURE 19. RCS of a stack of spherical scatterers. (a) Scatterers on the origin. (b) Scatterers on the off-centered location.

with each other. We denote the location of the lower scatterer in Figs. 18(a) and (b) by $\boldsymbol{r}_{1}$ and $\boldsymbol{r}_{2}$, respectively. Such a pair of point scatterers can be considered as an omni-directional scatterer with respect to the azimuthal angle $\alpha$, whereas the RCS has a directive pattern with respect to the zenith angle $\beta$. Thus, a stack of point scatterers serves as a simple example to show the effect of the local zenith angle $\beta(u)$ of an offcentered scatterer. Note that to reduce the effect of the scatter with nonzero altitude which is described in Section III-C, the vertical separation between the two point scatterers were set to $1 \mathrm{~cm}$ such that it is small compared to the wavelength. The reference point for the RCS computation is selected to the location of each lower scatterer, namely, $\boldsymbol{r}_{1}$ and $\boldsymbol{r}_{2}$.

Figs. 19(a) and (b) show the RCS derived from the simulated SAR images for the cases shown in Figs. 18(a) and (b), respectively. As shown in Fig. 19(a), the pair of scatterers at $\boldsymbol{r}_{1}(x=0)$ shows an omni-directional RCS pattern as the local zenith angle at the origin was unchanged with respect to the azimuthal angle. In contrast, when the stack of scatterers were moved to $\boldsymbol{r}_{2}(x=20 \mathrm{~cm})$ as depicted in Fig. 18(b), the resultant RCS was no longer omni-directional as shown in Fig. 19(b), because the local zenith angle depends on the azimuthal angle, as illustrated by the point $\boldsymbol{r}_{2}$ in Fig. 15. Therefore, one should be aware of the aforementioned dependency of the local zenith angle on the azimuthal angle in interpreting the RCS derived from a 2-D SAR or ISAR image. If this effect cannot be ignored, one should employ a 3-D imaging approach with a scanning surface which encloses the area to be imaged, as described in [13]. 


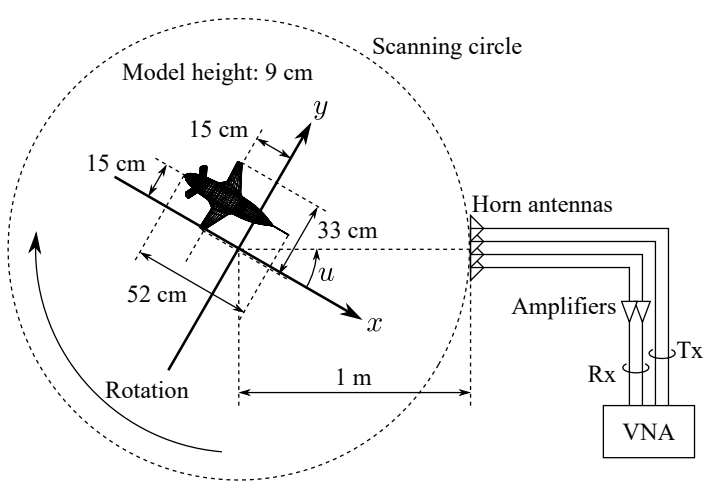

FIGURE 20. Experimental setup. The aircraft model was intentionally off-centered to demonstrate the effectiveness of the proposed correction factor. The four standard gain horn antennas were fixed, and the aircraft model was rotated.

\section{INDOOR MEASUREMENT}

In this section, we discuss the experimental validation carried out in an anechoic chamber. We employed an aircraft model made of aluminum for this measurement, and the improvement by the correction factor was investigated by comparing the measured result with the far-field RCS obtained from numerical electromagnetic simulations based on a multilevel fast multipole method (MLFMM) solver. Note that the data treated in this section is a part of the full dataset of cylindrical 3-D synthetic aperture measurement described in [13].

\section{A. OVERVIEW OF THE EXPERIMENT}

Figs. 20 and 21 show the setup and a photograph of this experiment, respectively; Table 2 lists the measurement parameters. In this experiment, a scaled aircraft model made of aluminum was used as a target, where the wingspan, the total length, and the height of the model were $33 \mathrm{~cm}, 52 \mathrm{~cm}$, and $9 \mathrm{~cm}$, respectively. As depicted in Fig. 20, the model was intentionally off-centered to demonstrate the effectiveness of the proposed correction factor; note that the similar approach was taken in Section III-B. This target was mounted on a styrene foam support on a turntable, as shown in Fig. 21.

Four standard gain horn antennas (Narda Model 640) which covers the frequency band from $8.2 \mathrm{GHz}$ to $12.4 \mathrm{GHz}$ were used as the transmitting and the receiving antennas. As shown in Fig. 21, two of them were horizontally polarized, and the other antennas were vertically polarized. The horizontal and the vertical separation of these antennas were approximately $11 \mathrm{~cm}$. The antenna altitude was set at the same altitude as the model such that $z_{0}=0$. As illustrated in Fig. 20, these antennas were connected to a vector network analyzer (VNA, Keysight N5242A) through microwave coaxial cables, where $30-\mathrm{dB}$ low noise amplifiers were inserted in the receiving paths. The antennas were fixed while the target was rotated using the turntable to create a circular aperture.

Table 2 lists the parameters of this indoor experiment. The center frequency and the bandwidth were $10.2 \mathrm{GHz}$ and $4 \mathrm{GHz}$; the measurement frequency was varied in steps of

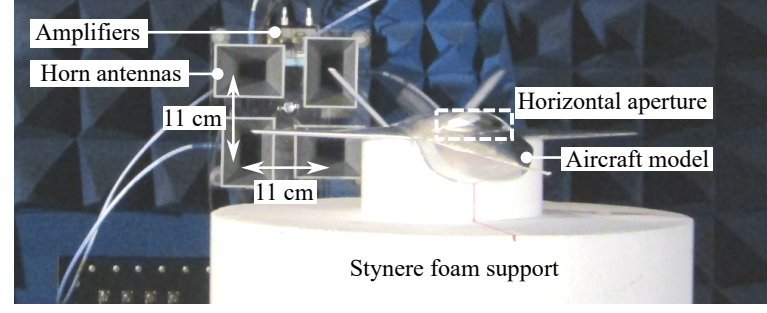

FIGURE 21. Photograph of the experiment. The aircraft model was mounted on a styrene foam cylinder. The horizontal and vertical separation of the antennas were $11 \mathrm{~cm}$. A horizontal aperture was at the front side of the model.

TABLE 2. Experimental parameters of the indoor measurement.

\begin{tabular}{l|l}
\hline Polarization & $\mathrm{HH}$ and VV \\
Center frequency & $10.2 \mathrm{GHz}$ \\
Bandwidth & $4 \mathrm{GHz}$ \\
Frequency step & $10 \mathrm{MHz}$ \\
Antenna scanning curve & Circle \\
Angular interval & {$\left[-180^{\circ}, 180^{\circ}\right]$} \\
Angular step & $0.8^{\circ}$ \\
Antenna altitude & $0.5 \mathrm{~m}$ \\
Radius of the scanning circle & $1.0 \mathrm{~m}$ \\
Number of points in a curve segment & $M=11$ \\
Order of the polynomial & $K=2$ \\
Scatterer & $\mathrm{Scaled}$ aircraft model \\
Pixel spacing & $4 \mathrm{~mm}$ \\
\hline
\end{tabular}

$10 \mathrm{MHz}$. We carried out full circular aperture measurement, where the azimuthal angle of the antenna was varied from $-180^{\circ}$ to $180^{\circ}$ in steps of $0.8^{\circ}$; the radius of the circular trajectory was $1 \mathrm{~m}$. In computing the numerical correction factor, number of points in a curve segment was $M=11$, and the order of the polynomial was $K=2$, which were same as the point scatterer simulation in Section III.

\section{B. EXPERIMENTAL RESULTS AND DISCUSSION}

Figs. 22(a) and (b) show the reconstructed spatial image of the aircraft model for $\mathrm{HH}$ and VV polarization. The proposed numerical correction factor was used to produce these images; note that quite similar images were obtained without the correction factor, namely, $g\left(k, \boldsymbol{r}_{0}, \boldsymbol{r}\right)=1$. Both images show the shape of the model clearly. Comparing Figs. 22(a) and (b), the VV-polarized image of Fig. 22(b) shows a bright response around $(x, y)=(-12,15) \mathrm{cm}$ (indicated by an arrow), which is unrecognizable in the HH-polarized image of Fig. 22(a). As shown in the photograph of Fig. 21 , this corresponds to the horizontal aperture (air-intake) of the aircraft model; because the backscattering intensity of a horizontal slit is maximized when a polarization vector is orthogonal to the length of the slit, the VV-polarized image was intensified at the area corresponds to the aperture on the aircraft model. As discussed below, the backscattering from this horizontal aperture significantly contributed to the measured RCS around $0^{\circ}$ in VV polarization.

Fig. 23 compares the reconstructed RCS from the HHpolarized spatial image, where (a) and (b) are without and with the correction factor, respectively. Because RCS of 


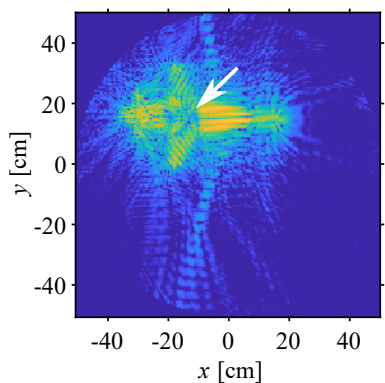

(a)

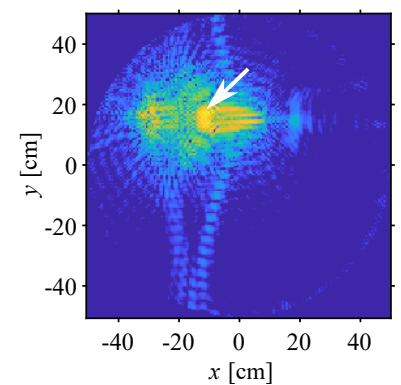

(b)
FIGURE 22. Spatial image of the aircraft model with the correction factor. An arrow indicates the location corresponding to a horizontal aperture of the model. (a) $\mathrm{HH}$ polarization. (b) VV polarization.

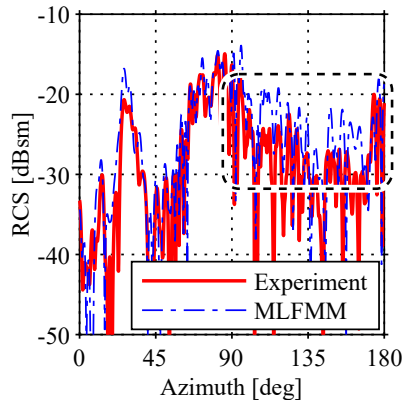

(a)

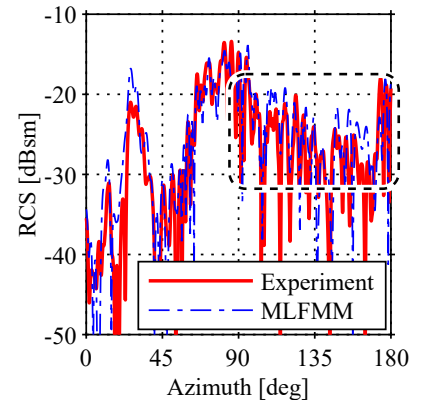

(b)
FIGURE 23. Reconstructed RCS (HH polarization). A dashed box indicates the range where the improvement by the correction factor is apparent. (a) Without correction factor. (b) With correction factor.

direct far-field measurement was unavailable owing to the spatial limitation of the anechoic chamber, we calculated the far-field RCS based on an MLFMM solver for comparison; the numerical result is also displayed in Fig. 23. Although the improvement by the correction factor is not as evident as the point scatterer simulation shown in Fig. 8, the improvement is certainly recognizable within the range of azimuthal angle from $90^{\circ}$ to $180^{\circ}$ (indicated by a dashed box in Fig. 23).

Fig. 24 shows the similar comparison as Fig. 23, where (a) and (b) are VV-polarized RCS without and with the correction factor, respectively. Like in the HH-polarized RCS shown in Fig. 23, the improvement by the correction factor is observed within the range of azimuthal angle from $90^{\circ}$ to $180^{\circ}$ (indicated by a dashed box in Fig. 24). Comparing Figs. 23 and 24 reveals that the VV-polarized RCS around $0^{\circ}$ is much stronger than the HH-polarized RCS. As mentioned earlier, this was attributed to the existence of the horizontal aperture in the aircraft model; that is, because a vertically polarized vector was orthogonal to the aperture length, the resultant backscattering from the aperture is more significant than those for a horizontally polarized wave.

From the aforementioned experimental results, we can conclude that the proposed correction factor is effective to

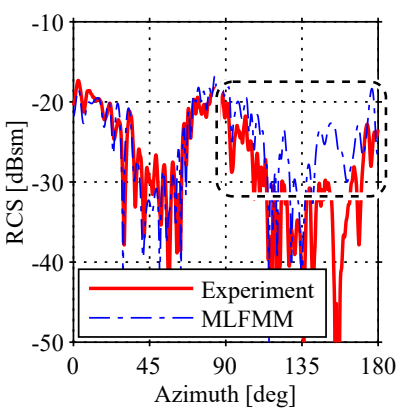

(a)

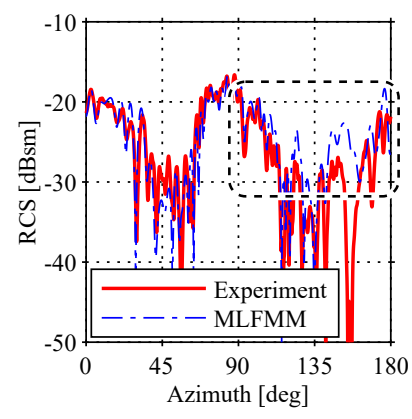

(b)
FIGURE 24. Reconstructed RCS (VV polarization). A dashed box indicates the range where the improvement by the correction factor is apparent. (a) Without correction factor. (b) With correction factor.

improve the far-field RCS prediction from near-field synthetic aperture imaging.

\section{AIRBORNE CIRCULAR SAR EXPERIMENT}

So far, we have proved the effectiveness of the proposed correction factor using the numerical simulations and the indoor measurement. In this section, we present alternative experimental validation of the proposed algorithm using a publicly available airborne CSAR dataset (GOTCHA) [26]. Because the flight trajectory of the aircraft fluctuates, this data can be used to test the applicability of the numerical correction factor, described in Section II-E, to the real antenna trajectory without analytical expression. Note that as the area to be imaged to the radar-carrying aircraft was sufficiently long, the improvement by the correction factor was not evident for this dataset. Therefore, the objective here is to demonstrate the applicability of the numerical correction factor, rather than proving the improvement by the correction factor, which was already accomplished by the numerical simulations in Section III and the indoor experiment in Section IV. Moreover, we explain a data-driven post-processing method to compensate for the antenna pattern which is unavailable from the publicly released dataset.

\section{A. OVERVIEW OF THE DATASET}

Table 3 lists the measurement parameters extracted from the dataset (we used "pass1" in the dataset). The center frequency, the bandwidth, and the frequency step were approximately $9.6 \mathrm{GHz}, 622 \mathrm{MHz}$, and $1.5 \mathrm{MHz}$, respectively. The antenna scanning curve was planned to be circular, but actually it strongly fluctuated due to the motion of the radar carrying aircraft, as shown in Fig. 25, where (a) shows the 3D plot of the aircraft trajectory, and (b) shows the variation of the radius of the trajectory with respect to the azimuthal angle. As the altitude and radius of the circular path varies, this dataset serves as a good example to show the applicability of the proposed numerical correction factor designed for an arbitrarily curved scanning trajectory. Using this CSAR data, we show the numerical correction factor can be calculated 
TABLE 3. Experimental parameters of the airborne CSAR measurement.

\begin{tabular}{l|l}
\hline Polarization & $\mathrm{HH}, \mathrm{HV}, \mathrm{VH}$, and VV \\
Center frequency & $9.6 \mathrm{GHz}$ \\
Bandwidth & $622 \mathrm{MHz}$ \\
Frequency step & $1.5 \mathrm{MHz}$ \\
Antenna scanning curve & Distorted circle \\
Angular interval & {$\left[-180^{\circ}, 180^{\circ}\right]$} \\
Angular step (average) & $8.5^{\circ} \times 10^{-3}$ \\
Antenna altitude (average) & $7266 \mathrm{~m}$ \\
Radius of the scanning circle (average) & $7265 \mathrm{~m}$ \\
Number of points in a curve segment & $M=1001$ \\
Order of the polynomial & $K=2$ \\
Scatterer & Small sphere \\
Pixel spacing & $1 \mathrm{~cm}$ \\
\hline
\end{tabular}

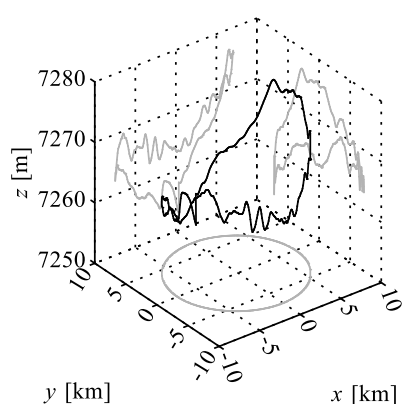

(a)

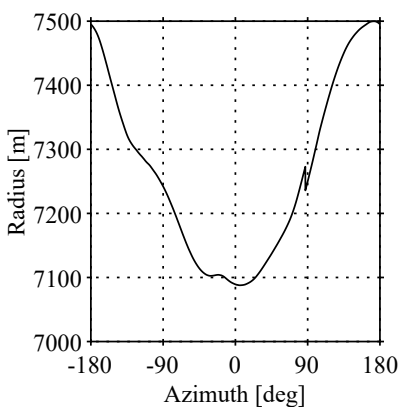

(b)
FIGURE 25. Aircraft trajectory and radius of the circular path. The abrupt changes in the radius around $90^{\circ}$ correspond to the start and end points of the circular path. (a) Trajectory. (b) Radius.

without suffering from any numerical instability.

Figs. 26(a) and (b) show an optical image and the reconstructed CSAR image, respectively. As information about the antenna used in this experiment was unavailable from the released dataset, the spatial images were reconstructed assuming an isotropic antenna pattern (i.e., $P\left(k, \boldsymbol{r}_{0}, \boldsymbol{r}\right)=1$ ). Moreover, a simple auto-focusing solution provided in the released dataset was used to improve the image reconstruction. In Fig. 26(b), we show the total power image $\psi_{T}(\boldsymbol{r})$ defined as follows:

$\psi_{T}(\boldsymbol{r})=\left|\psi_{H H}(\boldsymbol{r})\right|^{2}+\left|\psi_{H V}(\boldsymbol{r})\right|^{2}+\left|\psi_{V H}(\boldsymbol{r})\right|^{2}+\left|\psi_{V V}(\boldsymbol{r})\right|^{2}$

where $\psi_{q p}(\boldsymbol{r}), p, q \in\{H, V\}$ is the reconstructed spatial image for $q p$ polarization. As shown in Fig. 26(a), the lower half of the imaged area was occupied by the parking lot, where various passenger vehicles exist. In the upper left of the image, several calibration targets were deployed, including a trihedral, a dihedral, and a tophat reflector; these were clearly imaged in the CSAR image shown in Fig. 26(b).

\section{B. TARGETS OF INTEREST AND CALIBRATION}

In this section, we define the targets to be used for the demonstration, and describe the process of calibration. Figs. 27(a) and (b) show close-ups of the CSAR image around the simple targets and passenger vehicles (and a forklift) in the parking lot, respectively. Fig. 28 illustrates the geometry

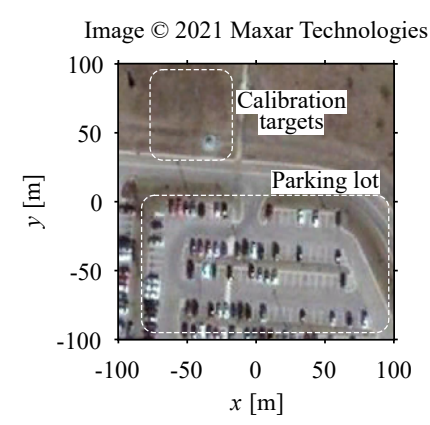

(a)

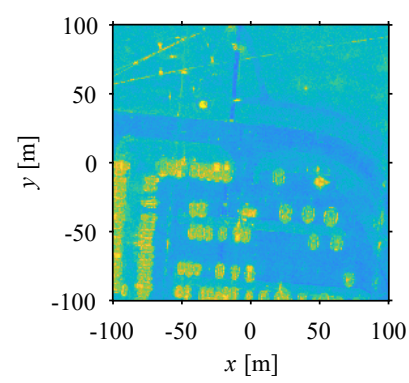

(b)
FIGURE 26. The Google Earth optical image and the airborne CSAR image.(a) Optical image. (b) SAR image (total power).

of these targets, and Table 4 summarizes their location, dimension, and heading. These simple targets were aligned such that the boresight direction of each target coincides the local zenith angle of $45^{\circ}$ at the target. The area shown in Fig. 27(a) contained triangular trihedral reflectors (indicated by "TR1" and "TR2"), and square dihedral reflectors (designated as "DR1" and "DR2"). The trihedral reflectors were expected to produce strong co-polarized (i.e., HH and VV) backscattering, whereas the cross-polarized (i.e., HV and $\mathrm{VH}$ ) backscattering from the dihedral reflectors were expected to be dominant because DR1 and DR2 were $45^{\circ}$ rotated about their boresight direction as illustrated in Fig. 28(b). The parking lot displayed in Fig. 27(b) contains the passenger vehicle (Nissan Sentra) labeled "PV1" to be examined. Moreover, the ground surfaces designated as "GS1" and "GS2" in Fig. 27 were used for estimating antenna pattern variation experienced at these locations, as discussed in detail in Section V-C.

According to the spatial filtering approach [18], [20] defined by Eqs. (51) and (52) in Section II-F, we applied a diskshaped window $w(r)$ defined in Eq. (51) to isolate the desired target, where the center of the window $\boldsymbol{r}_{r}$ was set the points corresponding to each target, and the radius of the window $a_{w}$ was selected to $1.5 \mathrm{~m}$ for the simple targets (i.e., TR1, TR2, DR1, and DR2), and $4.0 \mathrm{~m}$ for the passenger vehicle, as listed in Table 4; the appropriate radius of the window was determined by visual inspection of the reconstructed image shown in Fig. 27.

We used TR1 and DR1 to calibrate co- and cross-polarized RCS, based on the process defined in Eq. (50) in Section II-F. The local azimuthal angle $\alpha_{c}$ in Eq. (50) was selected to the corresponding heading angle listed in Table 4. Although the RCS was correctly calibrated at $\alpha_{c}$, the undesired variation of the unknown antenna pattern still remains in the calibrated RCS at this point, as we assumed an isotropic antenna pattern (i.e., $\left.P\left(k, \boldsymbol{r}_{0}, \boldsymbol{r}\right)=1\right)$ in the image reconstruction defined in Eq. (10). In Section V-C, we discuss how this unknown antenna pattern was estimated and compensated based on a data-driven post-processing scheme. 
TABLE 4. Location, dimension, heading, and window radius of the targets of interest.

\begin{tabular}{|c|c|c|c|c|c|}
\hline Label & Description & $(x, y, z)$-location $\left(\boldsymbol{r}_{r}\right)$ & Dimension & Heading & Window radius $\left(a_{w}\right)$ \\
\hline TR1 & Triangular trihedral & $(-28.0,38.2,-0.4) \mathrm{m}$ & $38.1 \mathrm{~cm}$ & $6.0^{\circ}$ & $1.5 \mathrm{~m}$ \\
\hline TR2 & Triangular trihedral for calibration & $(-32.6,42.1,-0.5) \mathrm{m}$ & $38.1 \mathrm{~cm}$ & $-81.5^{\circ}$ & $1.5 \mathrm{~m}$ \\
\hline DR1 & $45^{\circ}$-rotated square dihedral & $(-26.3,45.1,-0.4) \mathrm{m}$ & $30.5 \mathrm{~cm}$ & $9.0^{\circ}$ & $1.5 \mathrm{~m}$ \\
\hline DR2 & $45^{\circ}$-rotated square dihedral for calibration & $(-29.2,24.1,-0.5) \mathrm{m}$ & $30.5 \mathrm{~cm}$ & $88.5^{\circ}$ & $1.5 \mathrm{~m}$ \\
\hline PV1 & Passenger vehicle (Nissan Sentra) & $(22.7,-28.7,-0.2) \mathrm{m}$ & $\begin{array}{l}\text { Length: } 445 \mathrm{~cm} \\
\text { Width: } 171 \mathrm{~cm} \\
\text { Height: } 141 \mathrm{~cm}\end{array}$ & $86.2^{\circ}$ & $4.0 \mathrm{~m}$ \\
\hline GS1 & Ground surface near TR1 & $(-21.0,40.0,0.0) \mathrm{m}$ & Radius: $150 \mathrm{~cm}$ & N/A & $1.5 \mathrm{~m}$ \\
\hline GS2 & Ground surface near PV1 & $(36.0,-9.0,0.0) \mathrm{m}$ & Radius: $150 \mathrm{~cm}$ & N/A & $1.5 \mathrm{~m}$ \\
\hline
\end{tabular}

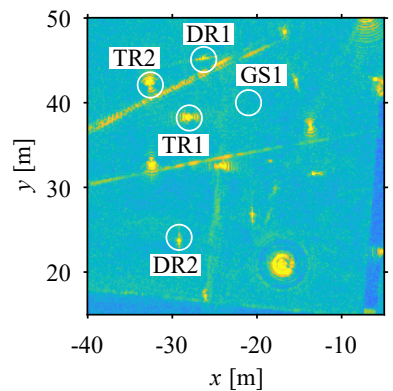

(a)

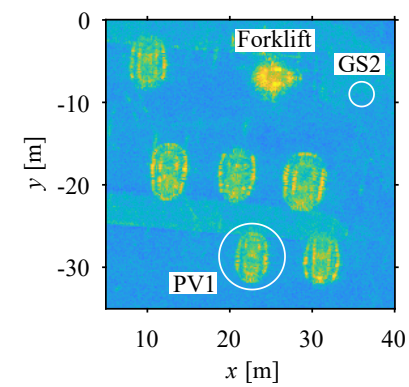

(b)
FIGURE 27. Close-ups of the SAR image. (a) Simple targets. (b) Passenger vehicles and forklift.

\section{ANTENNA PATTERN ESTIMATION AND COMPENSATION}

As mentioned in Section V-B, the RCS reconstructed from the CSAR images was affected by the antenna pattern variation. In this section, we propose a data-driven approach to estimate and compensate for the antenna pattern. From the optical image shown in Fig. 26(a), a flat lawn surface exists around the calibration targets (GR1) and in the parking lot (GR2). Because we can assume that the backscattering pattern from the ground surface exhibited azimuthal symmetry for all polarization channels, that is, $\mathrm{HH}, \mathrm{HV}, \mathrm{VH}$, and $\mathrm{VV}$, one could expect that the antenna pattern experienced at a ground surface can be estimated by fitting a curve to the RCS of the surface patch. The resultant curve was then used to subtract the antenna pattern variation of the targets near the ground. In the following discussion, we formulate the aforementioned algorithm.

Because the antenna trajectory was approximately circular, we can assume that the variation of the antenna pattern experienced at a certain image location can be modeled by a periodic function. Therefore, we consider modeling the antenna pattern variation by the following function:

$$
\bar{\sigma}_{\mathrm{dB}}(\alpha)=\sigma_{A} \cos \left(\alpha+\alpha_{\Delta}\right)+\sigma_{\Delta}
$$

where $\bar{\sigma}_{\mathrm{dB}}(\alpha)$ is the RCS of the in-scene target in $\mathrm{dB}$ scale at the local azimuthal angle $\alpha$, and $\sigma_{A}, \alpha_{\Delta}$, and $\sigma_{\Delta}$ are the amplitude, the initial phase, and the constant bias, respectively, which are to be determined.
We assume that we have $L$ discrete samples of the RCS in dB-scale computed via the algorithm described in Section II-G for an in-scene target exhibiting azimuthal symmetry (i.e., GR1 or GR2). We denote the local azimuthal angle of the $\ell$ th sample as $\alpha_{[\ell]}$ and the corresponding RCS sample as $\bar{\sigma}_{\mathrm{dB}[\ell]}=\bar{\sigma}_{\mathrm{dB}}\left(\alpha_{[\ell]}\right)$. According to Eq. (56), we consider the following representation of the $\ell$ th RCS sample:

$$
\begin{aligned}
\bar{\sigma}_{\mathrm{dB}[\ell]} & =\sigma_{A} \cos \left(\alpha_{[\ell]}+\alpha_{\Delta}\right)+\sigma_{\Delta} \\
& =\sigma_{C} \cos \alpha_{[\ell]}-\sigma_{S} \sin \alpha_{[\ell]}+\sigma_{\Delta} \\
\sigma_{C} & =\sigma_{A} \cos \alpha_{\Delta}, \quad \sigma_{S}=\sigma_{A} \sin \alpha_{\Delta}
\end{aligned}
$$

By considering Eq. (57) for all the RCS samples, we obtain the following matrix-vector notation:

$$
\begin{aligned}
\overline{\boldsymbol{\sigma}}_{\mathrm{dB}}= & \boldsymbol{M} \boldsymbol{\sigma} \\
\overline{\boldsymbol{\sigma}}_{\mathrm{dB}}= & {\left[\bar{\sigma}_{\mathrm{dB}[1]}, \bar{\sigma}_{\mathrm{dB}[2]}, \ldots, \bar{\sigma}_{\mathrm{dB}[\ell]}, \ldots, \bar{\sigma}_{\mathrm{dB}[L]}\right]^{T} } \\
\boldsymbol{M} & =\left[\begin{array}{ccc}
\cos \alpha_{[1]} & -\sin \alpha_{[1]} & 1 \\
\cos \alpha_{[2]} & -\sin \alpha_{[2]} & 1 \\
\vdots & \vdots & \vdots \\
\cos \alpha_{[\ell]} & -\sin \alpha_{[\ell]} & 1 \\
\vdots & \vdots & \vdots \\
\cos \alpha_{[L]} & -\sin \alpha_{[L]} & 1
\end{array}\right] \\
\boldsymbol{\sigma} & =\left[\begin{array}{ll}
\left.\sigma_{C}, \sigma_{S}, \sigma_{\Delta}\right]^{T}
\end{array}\right.
\end{aligned}
$$

where $\overline{\boldsymbol{\sigma}}_{\mathrm{dB}}$ is the data vector containing the measured RCS of an in-scene target, $\boldsymbol{M}$ is the known model matrix, and $\boldsymbol{\sigma}$ is an unknown vector containing the fitting coefficients to be determined. By employing the least-squares solution to Eq. (58a), the coefficient vector $\boldsymbol{\sigma}$ can be determined as follows:

$$
\boldsymbol{\sigma}=\left(\boldsymbol{M}^{T} \boldsymbol{M}\right)^{-1} \boldsymbol{M}^{T} \overline{\boldsymbol{\sigma}}_{\mathrm{dB}}
$$

Once the modified fitting coefficients $\sigma_{C}$, and $\sigma_{S}$ are determined, they can be converted into the original fitting coefficients, $\sigma_{A}$ and $\alpha_{\Delta}$, as follows:

$$
\begin{aligned}
\sigma_{A} & =\sqrt{\sigma_{C}^{2}+\sigma_{S}^{2}} \\
\alpha_{\Delta} & =\tan ^{-1}\left(\sigma_{S} / \sigma_{C}\right)
\end{aligned}
$$

Then, the determined antenna pattern $\bar{\sigma}_{\mathrm{dB}}(\alpha)$ is subtracted from the uncompensated RCS in $\mathrm{dB}$ scale, denoted by $\sigma_{\mathrm{dB}}(\alpha)$, which is assumed to be calibrated using a target of known RCS at a certain azimuthal angle $\alpha_{c}$, as described in 


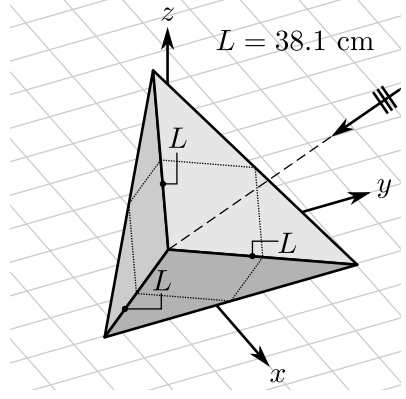

(a)

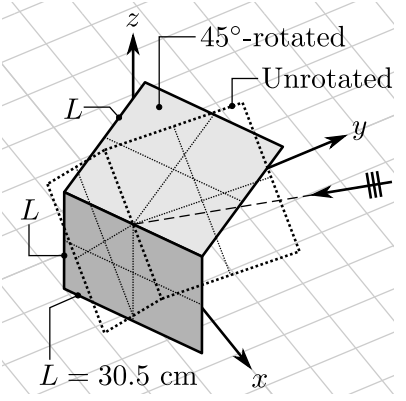

(b)

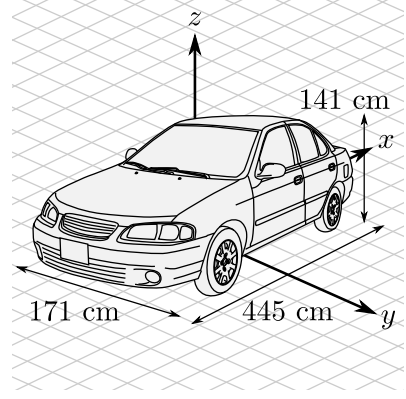

(c)

FIGURE 28. Geometry of the targets. (a) Trihedral reflector (TR1). (b) Dihedral reflector (DR1). (c) Passenger vehicle (PV1).

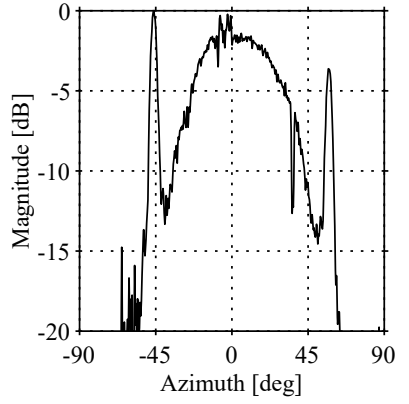

(a) (b)

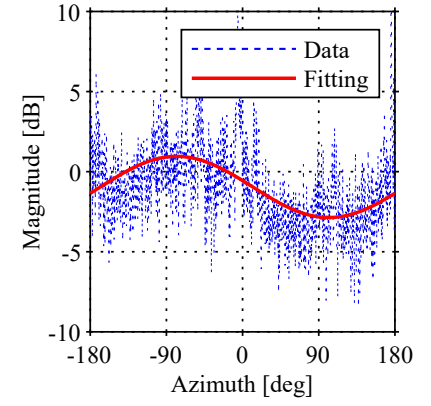

FIGURE 29. Uncompensated RCS of the trihedral normalized by its maximum value and antenna pattern variation estimated from the RCS of a image patch of vegetated ground. The RCS was asymmetric about heading angle $\left(6.0^{\circ}\right)$ owing to the antenna pattern variation. (a) Normalized RCS of the trihedral. (b) Antenna pattern.

Section II-F. Therefore, retaining the original RCS calibrated at $\alpha_{c}$, we compensate for the antenna pattern as follows:

$$
\widehat{\sigma}_{\mathrm{dB}}(\alpha)=\sigma_{\mathrm{dB}}(\alpha)-\bar{\sigma}_{\mathrm{dB}}(\alpha)+\bar{\sigma}_{\mathrm{dB}}\left(\alpha_{c}\right) .
$$

Fig. 29(a) shows the uncompensated RCS of the trihedral reflector (TR1), where the RCS was normalized by its maximum value for clarity of interpretation. Although this target was expected to exhibit a symmetric pattern with respect to its heading angle (i.e., $6^{\circ}$ ), the RCS shows asymmetric behavior as displayed in Fig. 29(a) owing to the antenna pattern variation. In fact, the peak on the right side in Fig. 29(a) (around $50^{\circ}$ ) is approximately 4 -dB lower than the peak on the left side (around $-45^{\circ}$ ). Fig. 29(b) shows the antenna pattern variation estimated from the RCS of a image patch of vegetated ground (GS1, see Fig. 27(a) and Table 4), based on Eq. (56) and Eq. (59); the RCS is normalized by its averaged value for displaying purpose. All RCS patterns obtained in this airborne CSAR experiment were compensated using the aforementioned procedure, and the results and discussion are given in Section V-D.

\section{RESULTS AND DISCUSSION}

In this section, we show the RCS derived form the airborne CSAR images. Figs. 30(a), (b), and (c) show the RCS derived from the CSAR images for the trihedral (TR1), the $45^{\circ}$ oriented dihedral (DR1), and the passenger vehicle (PV1), respectively. In Figs. 30(a) and (b), the simulated RCS using an MLFMM solver are also displayed, whereas the simulated RCS was unavailable for PV1 owing to limited computational resources and lack of a 3-D model for this target. Figs. 30(a) and (c) show the RCS for HH-polarization, whereas Fig. 30(b) shows the RCS for HV-polarization, because the cross-polarized backscattering was dominant for the $45^{\circ}$ oriented dihedral (DR1).

As shown in Figs. 30(a) and (b), excellent agreement is observed between the measured and the simulated RCS within a certain range of the azimuthal angle where the RCS is approximately greater greater than $0 \mathrm{dBsm}$. Otherwise, background clutter from the ground was dominant and the RCS pattern differs from the predicted values. As depicted in Fig. 30(c), the RCS of the passenger vehicle (PV1) exhibits four strong peaks at the azimuthal angle corresponding to the front, back, and sides of the vehicle. Note that we confirmed that quite similar results were obtained without the correction factor. Therefore, we can confirm that the proposed correction factor can be applied to the strongly fluctuating antenna trajectory without suffering any numerical instability.

\section{CONCLUSION}

In this research, we discuss the RCS measurement from synthetic aperture imaging with an arbitrary curved antenna scanning trajectory. For near-field measurement, the introduction of the correction factor is important to improve the far-field RCS prediction. We extend the previously developed correction factor for 3-D synthetic aperture imaging with 2-D antenna scanning along an arbitrary surface to a 1-D scanning case with an arbitrary curved trajectory. The improvement by the proposed correction factor was proved by the numerical simulations and the indoor experiment. To show the applicability of the algorithm to a curved trajectory without its analytical representation, we apply the proposed technique to the airborne CSAR data. Although the improvement is not evident in this case because the distance from the antenna to the targets are sufficiently long, we confirm that the application of the numerical correction factor does not introduce any adverse effect on the RCS prediction, implying that 


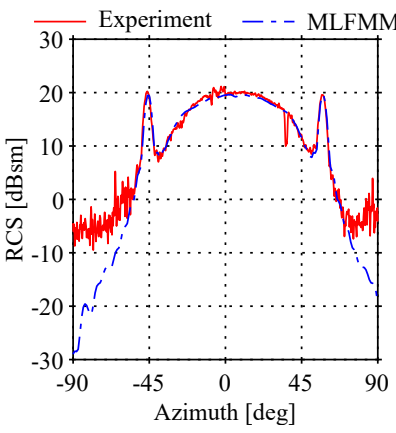

(a)

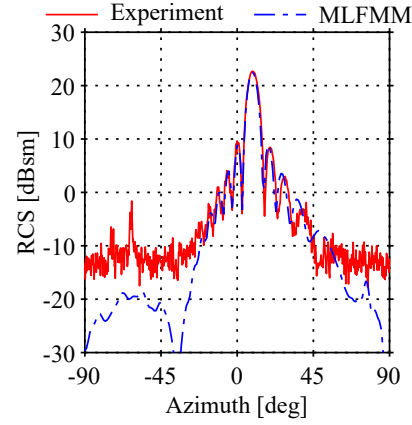

(b)

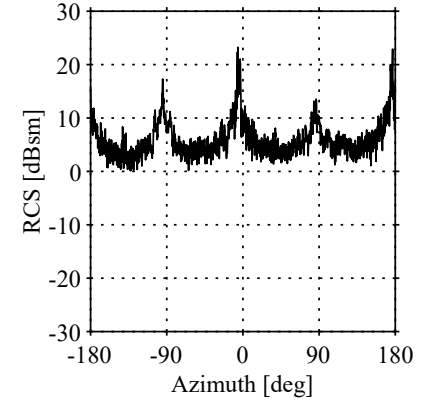

(c)

FIGURE 30. RCS derived from the CSAR image with the correction factor. Results of MLFMM simulation for PV1 was unavailable because of the limited computational resource and the lack of a 3-D model for this target. (a) TR1 (HH polarization). (b) DR1 (HV polarization). (c) PV1 (HH polarization).

the method can be utilized for unstably fluctuating antenna scanning trajectory without suffering from any numerical instability. In this CSAR experiment, we also show that the antenna pattern variation can be compensated without having its knowledge using a data-driven post-processing approach.

In future works, we plan to prove the effectiveness of the proposed correction factor for outdoor measurement with a SAR antenna mounted on an unstable platform such as a vehicle or a ship. This type of measurement is useful for RCS measurement of a very large target for which indoor measurement in an anechoic chamber is infeasible owing to the spatial limitations.

\section{ACKNOWLEDGMENTS}

The authors would like to thank the Air Force Research Laboratory (AFRL) for providing the airborne circular SAR datasets (GOTCHA) [26].

\section{REFERENCES}

[1] D. G. Falconer, "Extrapolation of near-field RCS measurements to the far zone," IEEE Trans. Antennas Propag., vol. 36, no. 6, pp. 822-829, Jun. 1988.

[2] G. Schnattinger, R. A. M. Mauermayer, and T. F. Eibert, "Monostatic Radar Cross Section Near-Field Far-Field Transformations by Multilevel Plane-Wave Decomposition," IEEE Trans. Antennas Propag., vol. 62, no. 8, pp. 4259-4268, Aug. 2014.

[3] O. Neitz, R. A. M. Mauermayer, and T. F. Eibert, "3-D Monostatic RCS Determination From Multistatic Near-Field Measurements by Plane-Wave Field Synthesis," IEEE Trans. Antennas Propag., vol. 67, no. 5, pp. 33873396, May 2019.

[4] S. Omi, M. Hirose, M. Ameya, and S. Kurokawa, "Plane-Wave Synthesis Employing Propagating Plane-Wave Expansion for Three- and TwoDimensional RCS Prediction Including the Multiple Scattering Effects," IEEE Trans. Antennas Propag., Early Access.

[5] J. W. Odendaal and J. Joubert, "Radar cross section measurements using near-field radar imaging," IEEE Trans. Instrum. Meas., vol. 45, no. 6, pp. 948-954, Dec. 1996.

[6] A. Broquetas, J. Palau, L. Jofre, and A. Cardama, "Spherical wave nearfield imaging and radar cross-section measurement," IEEE Trans. Antennas Propag., vol. 46, no. 5, pp. 730-735, May 1998.

[7] T. Vaupel and T .F. Eibert, "Comparison and application of near-field ISAR imaging techniques for far-field radar cross section determination," IEEE Trans. Antennas Propag., vol. 54, no. 1, pp. 144-151, Jan. 2006.

[8] A. Osipov, H. Kobayashi, and H. Suzuki, "An Improved Image-Based Circular Near-Field-to-Far-Field Transformation," IEEE Trans. Antennas Propag., vol. 61, no. 2, pp. 989-993, Feb. 2013.
[9] T. Vaupel and F. Weinmann, "Validation of a 3-D near-field ISAR imaging technique with far-field RCS extraction by means of a hybrid GO-PO/PTD ray tracing algorithm," in 2009 3rd European Conference on Antennas and Propagation, Mar. 2009, pp. 691-695.

[10] L. Pu, X. Zhang, J. Shi, S. Wei, L. Li, and X. Tang, "Three Dimensional Image-Based Radar Cross Section Extrapolation via Planar Projective Transforms," IEEE Access, vol. 7, pp. 138990-139000, 2019.

[11] T. Vaupel, "An improved 3-D near-field ISAR imaging technique with extended far-field RCS extraction," in 2015 IEEE International Symposium on Antennas and Propagation USNC/URSI National Radio Science Meeting, Jul. 2015, pp. 699-700.

[12] T. Vaupel, "A Fast 3-D Cylindrical Scanning Near-Field ISAR Imaging Approach With Extended Far-Field RCS Extraction Based on a Modified Focusing Operator," in Proceedings of EUSAR 2016: 11th European Conference on Synthetic Aperture Radar, Jun. 2016, pp. 97-101.

[13] T. Watanabe and H. Yamada, "Far-Field Radar Cross-Section Determination From Near-Field 3-D Synthetic Aperture Imaging With Arbitrary Antenna Scanning Surfaces," 2021, [Online]. Available: 10.36227/techrxiv.16802419.

[14] I. J. LaHaie, "Overview of an image-based technique for predicting farfield radar cross section from near-field measurements," IEEE Antennas and Propagation Magazine, vol. 45, no. 6, pp. 159-169, Dec. 2003.

[15] I. J., LaHaie, "An improved version of the circular near field-to-far-field transformation (CNFFFT)," Proceedings of the 27th Annual Meeting of the Antenna Measurement Techniques Association (AMTA'05), pp. 196-201, Newport, RI, 2005.

[16] D. L. Mensa, High Resolution Radar Imaging, Artech House, 1983.

[17] M. Soumekh, Fourier Array Imaging, Englewood Cliffs, NJ: Prentice Hall, 1994.

[18] M. Soumekh, Synthetic Aperture Radar Signal Processing: with MATLAB Algorithms, John Wiley \& Sons, 1999.

[19] M. Cheney and B. Borden, Fundamentals of Radar Imaging, SIAM, 2009

[20] T. Watanabe, H. Yamada, Y. Tokutake, R. Sato, and Y. Yamaguchi, "Retrieval of polarimetric azimuthal angular characteristics via the application of target decomposition to spectral domain circular SAR images," IEEE Trans. Geosci. Remote Sens., vol. 57, no. 5, pp. 2963-2982, May 2019.

[21] M. Soumekh, "Reconnaissance with slant plane circular SAR imaging,", IEEE Trans. Image Proc., vol. 5, no. 8, pp. 1252-1265, Aug. 1996.

[22] A. Ishimaru, T.-K. Chan, Y. Kuga, "An imaging technique using confocal circular synthetic aperture radar," IEEE Trans. Geosci. Remote Sens., vol. 36, no. 5, pp. 1524-1530, September 1998.

[23] P. O. Frolind, A. Gustavsson, M. Lundberg, and L. M. H. Ulander, "Circular-aperture VHF-band synthetic aperture radar for detection of vehicles in forest concealment," IEEE Trans. Geosci. Remote Sens., vol. 50, no. 4, pp. 1329-1339, Apr. 2012.

[24] O. Ponce, P. Prats-Iraola, M. Pinheiro, M. Rodriguez-Cassola, R. Scheiber A. Reigber, and A. Moreira, "Fully polarimetric high-resolution 3-D imaging with circular SAR at L-band," IEEE Trans. Geosci. Remote Sens., vol. 52, no. 6. pp. 3074-3090, June 2014.

[25] O. Ponce, P. Prats-Iraola, R. Scheiber, A. Reigber, and A. Moreira, "First airborne demonstration of holographic SAR tomography with fully polarimetric multicircular acquisitions at L-band," IEEE Trans. Geosci. Remote Sens., vol. 54, no. 10. pp. 6170-6196, Oct. 2016. 
[26] E. Ertin, C. D. Austin, S. Sharma, R. L. Moses, and L. C. Potter, "GOTCHA experience report: three-dimensional SAR imaging with complete circular apertures," Orlando, Florida, USA, Apr. 2007, p. 656802.

[27] G. T. Ruck, D. E. Barrick, W. D. Stuart, and C. K. Krichbaum, Radar Cross Section Handbook, Vol. 1, Plenum Press, New York, 1970.

[28] C. A. Balanis, Antenna Theory: Analysis and Design, 4th edition, Wiley, 2016.

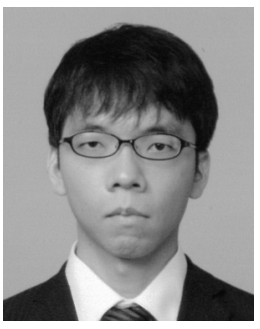

TAKUMA WATANABE (Member, IEEE) received B.E., M.E., and Dr. Eng. degrees in electrical and information engineering from Niigata University, Niigata, Japan, in 2010, 2012, and 2015, respectively.

From 2015 to 2017, he was with the Central Research Institute of Electric Power Industry, Yokosuka, Japan. From 2017 to 2018, he was with PASCO Corporation, Tokyo, Japan. Since 2018, he has been with Fujitsu Ltd., Kawasaki, Japan, where he is a research scientist at an inhouse laboratory. His research interests include image reconstruction problems, synthetic aperture radar (SAR) signal processing, radar cross-section (RCS) measurement, array signal processing, and electromagnetic scattering problems.

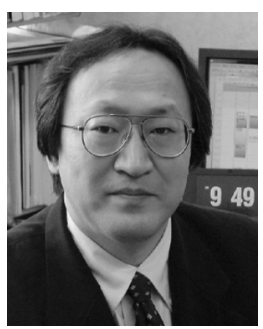

HIROYOSHI YAMADA (Member, IEEE) received B.E., M.E., and Dr. Eng. degrees in electronic engineering from Hokkaido University, Sapporo, Japan, in 1988, 1990, and 1993, respectively.

Since 1993, he has been a member of the Faculty of Engineering, Niigata University, Niigata, Japan, where he is currently a professor. From 2000 to 2001, he was a visiting scientist at the Jet Propulsion Laboratory, California Institute of Technology, Pasadena, CA, USA. His research interests include array signal processing, polarimetric radar interferometry, and high-resolution techniques. 